\title{
Produits tensoriels continus d'espaces et d'algèbres de Banach
}

\author{
A. Guichardet \\ Université de Poitiers, Faculté des Sciences
}

Reçu le ler Mars, 1967

\begin{abstract}
The notion of tensor product of a family $\left(A_{i}\right)_{\imath} \in I$ of Banach algebras is generalized to the case when $I$ is a topological space; in this case $\hat{\otimes} A_{i}$ is generated by some elements $\otimes x_{i}$, the family $\left(x_{i}\right)$ being subjected to certain conditions: for instance the function $i \rightarrow \mid x_{i} \|$ must be continuous. This notion is applied to Quantum Field Theory in the following sense: certain algebras of observables can be considered as continuous tensor products of simpler ones, namely of algebras of observables with one degree of freedom.
\end{abstract}

\section{Table des matières}

$\S 1$. Rappels sur les produits tensoriels finis d'espaces de Banach . . . . . 263

§. Produits tensoriels infinis d'espaces de Banach . . . . . . . . . . . 264

§3. Produits tensoriels infinis d'algèbres de Banach unitaires . . . . . . . 266

$\$ 4$. Produits continus de nombres complexes . . . . . . . . . . . . . . 267

$\S 5$. Définition des produits tensoriels continus d'espaces de Banach . . . . 268

§. Associativité du produit tensoriel continu. . . . . . . . . . . . . . 271

$\S 7$. Produits tensoriels continus d'applications linéaires continues . . . . . 275

$\$ 8$. Produits tensoriels continus d'espaces $L^{1}$. . . . . . . . . . . . . . 278

$\S 9$. Produit tensoriel inductif d'une famille continue constante d'espaces de

Banach . . . . . . . . . . . . . . . . . . 280

$\S 10$. Produits tensoriels continus d'algèbres de Banach unitaires . . . . . . 281

$\$$ 11. Produits tensoriels continus de certaines familles d'espaces hilbertiens . 283

$\$ 12$. Produits tensoriels continus de $C^{*}$-algèbres; applications à la théorie quantique des champs . . . . . . . . . . . . . . . . 284

Bibliographie . . . . . . . . . . . . . . . . . 287

\section{Introduction}

De même que le produit tensoriel $E_{1} \hat{\otimes} E_{2}$ de deux espaces de Banach est engendré par certains éléments $x_{1} \otimes x_{2}$, le produit tensoriel d'une «fàmille continue» d'espaces de Banach $\left(E_{i}\right)_{i} \in_{I}$ est engendré par certains éléments notés $\otimes x_{i}$; rnais ici la famille $\left(x_{i}\right)$ n'est pas un élément quelconque de $\Pi E_{i}$, elle est soumise à certaines conditions dites de «continuité», autrement dit elle doit appartenir à un certain sous-ensemble $\Gamma$ de $\Pi E_{i}$ qu'on définit axiomatiquement ( $\$ 5$ ), d'une façon assez analogue à ce qui se fait pour définir les champs mesurables et surtout les champs 
continus d'espaces de Banach (cf. [2]). L'espace $\hat{\otimes} E_{i}$, produit tensoriel continu des $E_{i}$, est alors construit, tout comme dans le cas de deux espaces, comme quotient d'un espace $L^{1}(\$ 5)$. Dans le cas de deux espaces, les éléments $x_{1} \otimes x_{2}$ jouissent de deux propriétés remarquables: $d^{\prime}$ une part $\lambda_{1} x_{1} \otimes \lambda_{2} x_{2}=\lambda_{1} \lambda_{2} x_{1} \otimes x_{2}$ ( $\lambda_{1}$ et $\lambda_{2}$ scalaires), et d'autre part la distributivité par rapport à l'addition; on parvient à généraliser la première propriété en définissant au préalable (\$4) le produit $\overparen{\Pi} \lambda_{i}$ d'une famille continue de nombres complexes: pour cela on se donne une mesuro positive $\mu$ sur $I$ et on pose $\overparen{\Pi} \lambda_{i}=\exp \left(\int \log \lambda_{\imath} d \mu(i)\right)$, ce qui ne peut se faire qu'en imposant des conditions très restrictives à $I$ et $\mu$. Par contre, malheureusement, on ne voit pas comment généraliser la distributivité: $\otimes\left(x_{i}+y_{i}\right)=\cdots$. Le produit tensoriel continu jouit d'un certain nombre de propriétés attendues: citons par exemple la relation $\left\|\otimes x_{\imath}\right\|=\overparen{\Pi}\left\|x_{i}\right\|$ (prop. 7), deux propriétés d'associativité (prop. 8 et 9), une propriété de "permutabilité» avec les quotients (prop. 11), le fait que certains produits tensoriels continus d'espaces $L^{1}$ sont encore des cspaces $L^{1}$ (prop. 12) ; par contre un phénomène assez inattendu apparaît lorsque l'on définit les produits tensoriels continus $\hat{\otimes} T_{i}$ d'applications linéaires continues: $\hat{\otimes} T_{i}$ peut être nul sans qu'aucun $T_{i}$ le soit (remarque 5).

La définition des produits tensoriels continus d'algèbres de Banach unitaires ne présente aucune difficulté $(\$ 10)$; quant aux $C^{*}$-algèbres, on définit formellement la $C^{*}$-algèbre produit tensoriel continu comme $C^{*}$-algèbre enveloppante de $\hat{\otimes} A_{i}(\S 12)$, mais on n'en connaît que peu de propriétés, faute de savoir définir en toute généralité les produits tensoriels continus d'espaces hilbertiens (\$11); on espère malgré tout que, convenablement améliorée, la théorie des produits tensoriels continus de $C^{*}$-algèbres pourra être utile à la théorie quantique des champs, par exemple pour construire un modèle de l'axiomatique de HAAG-Kastren, ou encore pour traiter les représentations des relations de commutation correspondant à une famille continue d'indices (cf. fin du $\S 12$ ), de la même façon que les produits tensoriels infinis sont utiles pour les représentations des relations de commutation ou d'anticommutation correspondant à une famille discrète d'indices (cf. [3]).

\section{$\$ 1$. Rappels sur les produits tensoriels finis d'espaces de Banach}

Soit $\left(E_{i}\right)_{i \in I}$ une famille finie d'espaces de Banach complexes; notons $v$ la mesure positive atomique sur $\Pi E_{i}$ attribuant à tout point $x=\left(x_{i}\right)$ de $\Pi E_{i}$ la masse $v(x)=\Pi\left\|x_{i}\right\|$; notons $\mathscr{K}\left(\Pi E_{i}\right)$ l'ensemble des fonctions complexes $F$ sur $\Pi E_{i}$ telles que $F(x)=0$ sauf pour un nombre fini de $x$; on notera en particulier $\delta_{x}$ la fonction égale à 1 en $x$ et à 0 ailleurs; on 
définit sur $\mathscr{K}\left(\Pi E_{i}\right)$ une semi-norme par

$$
\|F\|=\sum_{x \in \Pi E_{i}}|F(x)| v(x)
$$

l'espace séparé-complété de $\mathscr{K}\left(\Pi E_{i}\right)$ pour cette semi-norme est $L^{1}\left(\Pi E_{i}\right.$, $\nu)$. Soit $M$ le sous-espace vectoriel de $\mathscr{K}\left(\Pi E_{i}\right)$ engendré par les éléments de la forme $\delta_{\left(\lambda_{i} x_{i}\right)}-\Pi \lambda_{i} \cdot \delta_{\left(x_{i}\right)}$ où $x_{i} \in E_{i}$ et $\lambda_{i} \in \mathbb{C}$; ou de la forme $\mid \delta_{\left(x_{i}\right)}+\delta_{\left(y_{i}\right)}-\delta_{\left(z_{i}\right)}$ où $x_{i}=y_{i}=z_{i}$ sauf pour un indice $i_{0}$ et $z_{i_{0}}=x_{i_{0}}+y_{i_{0}}$; soit $\bar{M}$ l'adhérence de son image canonique dans $L^{1}\left(\Pi E_{i}, v\right)$. L'espace quotient $L^{1}\left(\Pi E_{i}, v\right) / \bar{M}$ est le produit tensoriel projectif de la famille $\left(E_{i}\right)$; nous le noterons ici $\widehat{\bigotimes}_{i \in I} E_{i}$ ou plus simplement $\widehat{\otimes} E_{i}$; on note $\otimes x_{i}$ l'image canonique de $\delta_{\left(x_{i}\right)}$ et on montre que $\left\|\otimes x_{i}\right\|=\Pi\left\|x_{i}\right\|$. On peut encore définir $\widehat{\otimes} E_{i}$ comme le complété de $\mathscr{K}\left(\Pi E_{i}\right) / M$ pour la semi-norme quotient, qui est en fait une norme.

Le produit tensoriel $\hat{\otimes} E_{i}$ et l'application multilinéaire canonique $u$ : $\Pi E_{i} \rightarrow \hat{\otimes} E_{i}$ jouissent de la propriété universelle suivante: si $E$ est un espace de Banach, en associant a toute application linéaire continue $v$ de $\hat{\otimes} E_{i}$ dans $E$ l'application multilinéaire $v \cdot u$, on obtient un isomorphisme isométrique del'espace de Banach $\mathscr{L}\left(\hat{\otimes} E_{i}, E\right)$ sur l'espace de Banach des applications multilinéaires continues $\Pi E_{i} \rightarrow E$.

Signalons encore les propriétés suivantes, dont la première justifie le nom de produit tensoriel projectif: soit, pour tout $i, F_{i}$ un sous-espace vectoriel de $E_{i}$ et soit $F$ le sous-espace vectoriel de $\hat{\otimes} E_{i}$ engendré par les éléments $\otimes x_{i}$ où $x_{i} \in F_{i}$ pour au moins un indice $i$; alors il existe un isomorphisme isométrique de $\hat{\otimes} E_{i} \mid \bar{F}$ sur $\hat{\otimes}\left(E_{i} \mid \bar{F}_{i}\right)$ transformant $\dot{\otimes}_{\otimes}$ en $\otimes \dot{x}_{i}$ pour tout $\left(x_{i}\right) \in \Pi E_{i}$. Propriété d'associativité : pour toute partition $I=\bigcup_{a \in A} I_{a}$, il existe un isomorphisme isométrique de $\bigotimes_{i \in I} E_{i}$ sur $\hat{\bigotimes}_{a \in A}\left(\hat{\bigotimes}_{i \in I_{a}} E_{i}\right)$ transformant tout élément $\bigotimes_{i \in I} x_{i}$ en $\bigotimes_{a \in A}\left(\bigotimes_{i \in I_{a}} x_{i}\right)$.

\section{\$ 2. Produits tensoriels infinis d'espaces de Banach}

Soit $\left(E_{i}\right)_{i \in I}$ une famille d'espaces de Banach complexes et soit, pour tout $i, \xi_{i}$ un élément de norme 1 de $E_{i}$. Soit $\Gamma$ le sous-ensemble de $\Pi E_{i}$ formé des familles $\left(x_{i}\right)$ telles que $x_{i}=\xi_{i}$ pour presque tout $i$ (i. e. sauf pour un nombre fini de $i$ ); soit $v$ la mesure positive atomique sur $\Gamma$ attribuant à tout élément $x=\left(x_{i}\right)$ de $\Gamma$ la masse $\nu(x)=\Pi\left\|x_{i}\right\|$; on définit $\mathscr{K}(\Gamma)$ et $L^{1}(\Gamma, v)$ comme au $\S 1$; même chose pour $M$, où l'on suppose en outre $\lambda_{i}=1$ pour presque tout $i$, puis pour $\bar{M}$; l'espace quotient $L^{1}(\Gamma, v) \mid \bar{M}$ sera noté $\hat{\bigotimes}_{i \in I}^{\left(\xi_{i}\right)} E_{i}$ ou plus simplement $\hat{\otimes}^{\xi} E_{i}$; pour tout $\left(x_{i}\right) \in \Gamma$ on note encore $\otimes x_{i}$ l'image canonique de $\delta_{\left(x_{i}\right)}$ dans $\hat{\otimes}^{\xi} E_{i}$; on voit donc que les éléments $\otimes x_{i}$ forment une partie totale de $\hat{\otimes}^{\xi} E_{i}$. 
Mais on peut aussi définir $\hat{\otimes}^{\xi} E_{i}$ comme limite inductive des produits tensoriels finis; plus précisément, en utilisant la propriété d'associativité du $\S 1$, on voit facilement que les produits tensoriels finis $\underset{i \in J}{\otimes} E_{i}(J$ partie finie de $I$ ) forment un systeme inductif avec des morphismes isométriques:

$$
\begin{array}{ll}
\bigotimes_{i \in J} E_{i} & \rightarrow \hat{\bigotimes}_{i \in K} E_{i} \quad(J \subset K) \\
\bigotimes_{i \in J} x_{i} & \rightarrow \bigotimes_{i \in K} y_{i}
\end{array}
$$

où $y_{i}=x_{i}$ ou $\xi_{i}$ suivant que $i \in J$ ou $i \in K-J$. D'autre part $L^{1}(\Gamma, v)$ apparaît comme la limite inductive des $L^{1}\left(\prod_{i \in J} E_{i}, v_{J}\right)$ et $\bar{M}$ comme celle des $\bar{M}_{J} ;$ il en résulte que

Proposition 1. Il existe un isomorphisme isométrique de la limite inductive du système inductif des produits tensoriels finis $\hat{\bigotimes}_{i \in J} E_{i}$ sur le produit tensoriel infini $\hat{\bigotimes}_{i \in I}^{\xi} E_{i}$ transformant, pour tout élément $\bigotimes_{i \in J} x_{i}$, son image canonique dans $\lim _{i \in J} \hat{\otimes} E_{i}$ en l'élément $\bigotimes_{i \in I} y_{i}$ où $y_{i}=x_{i}$ pour $i \in J$ et $y_{i}$ $=\xi_{i}$ pour $i \in I-J$.

Corollaire 1. Pour tout $\left(x_{i}\right) \in \Gamma$ on a $\left\|\otimes x_{i}\right\|=\Pi\left\|x_{i}\right\|$.

Proposition 2. Soit, pour tout $i \in I, F_{i}$ un sous-espace vectoriel de $E_{i}$; on note $\dot{x}_{i}$ l'image canonique dans $E_{i} \mid \bar{F}_{i}$ d'un élément quelconque $x_{\imath}$ de $E_{i}$ et on suppose $\left\|\dot{\xi}_{i}\right\|=1$; soit $F$ le sous-espace vectoriel des $\hat{\otimes}^{\xi} E_{i}$ engendré par les éléments $\otimes x_{i}$ où $x_{i} \in F_{i}$ pour au moins un $i$; alors il existe un isomorphisme isométrique de $\left(\hat{\otimes}^{\left(\xi_{i}\right)} E_{i}\right) \mid \bar{F}$ sur $\hat{\otimes}^{\left(\dot{\xi}_{i}\right)}\left(E_{i} \mid \bar{F}_{i}\right)$ transformant $\dot{\widehat{\otimes x}}_{i}$ en $\otimes \dot{x}_{i}$ pour tout $\left(x_{i}\right) \in \Gamma$.

Notons $\Gamma^{0}$ et $v^{0}$ les éléments associés à $\left(E_{i} \mid \bar{F}_{i}\right)$ et $\left(\dot{\xi}_{i}\right)$ comme $\Gamma$ et $v$ sont associés à $\left(E_{i}\right)$ et $\left(\xi_{i}\right)$; l'application multilinéaire continue $\left(x_{i}\right) \rightarrow \otimes \dot{x}_{i}$ de $\Gamma$ dans $\hat{\otimes}^{\left(\dot{\xi}_{i}\right)}\left(E_{i} / \bar{F}_{i}\right)$ définit une application linéaire continue de $\hat{\otimes}^{\left(\xi_{i}\right)} E_{i}$ dans $\hat{\otimes}^{\left(\dot{\xi}_{i}\right)}\left(E_{i} \mid \bar{F}_{i}\right)$, nulle sur $F$, d'où une application linéaire continue

$$
u:\left(\hat{\otimes}^{\left(\xi_{i}\right)} E_{i}\right) \mid \bar{F} \rightarrow \hat{\otimes}^{\left(\dot{\xi}_{i}\right)}\left(E_{i} \mid \bar{F}_{i}\right)
$$

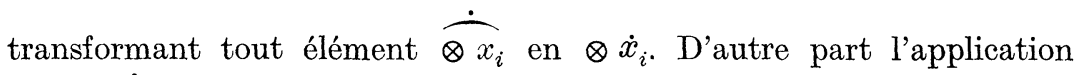

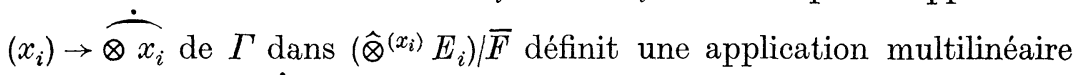

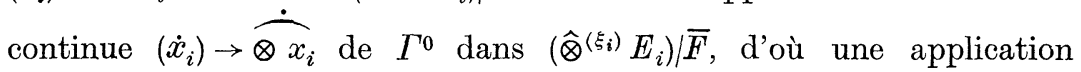
linéaire continue

$$
v: \hat{\otimes}^{\left(\dot{\xi}_{i}\right)}\left(E_{i} / \bar{F}_{i}\right) \rightarrow\left(\hat{\otimes}^{\left(\xi_{i}\right)} E_{i}\right) / \bar{F} ;
$$

enfin il est facile de voir que $u$ et $v$ sont isométriques et réciproques. 
Proposition 3. Soient, pour tout $i \in I, X_{i}$ un ensemble, $v_{i}$ une mesure positive atomique sur $X_{i}$ attribuant $\dot{a}$ chaque point $\chi_{i} \in X_{i}$ une masse $\nu_{i}\left(\chi_{i}\right), \omega_{i}$ un point de $X_{i}$ de masse 1 ; soient $X$ le sous-ensemble de $\Pi X_{i}$ formé des familles $\chi=\left(\chi_{i}\right)$ telles que $\chi_{i}=\omega_{i}$ pour presque tout $i$ et $v$ la mesure positive sur $X$ attribuant à tout point $\chi$ la masse $\Pi v_{i}\left(\chi_{i}\right)$. Il existe un isomorphisme isométrique de $\hat{\otimes}^{\left(\delta_{\omega_{i}}\right)} L^{1}\left(X_{i}, v_{i}\right)$ sur $L^{1}(X, v)$ transformant tout élément $\otimes f_{i}$ en la fonction $\chi \rightarrow \Pi f_{i}\left(\chi_{i}\right)$.

La démonstration, à l'aide des limites inductives et du résultat (classique) dans le cas des produits finis, est facile et laissée au lecteur.

Remarque 1. On reprend les notations du début du $\S$; soit, pour tout $i, \varphi_{i}$ une forme linéaire continue sur $E_{i}$ de façon que $\varphi_{i}\left(\xi_{i}\right)=1$ pour presque tout $i$ et que le produit $\Pi\left\|\phi_{i}\right\|$ soit convergent; pour tout $x=\left(x_{i}\right)$ $\in \Gamma$ le produit $I I \varphi_{i}\left(x_{i}\right)$ a un sens puisque pour presque tout $i$ on a $\varphi_{i}\left(x_{i}\right)=\varphi_{i}\left(\xi_{i}\right)=1$; pour tout $F \in L^{1}(T, v)$ on a

$$
\sum_{x \in T^{\prime}}|F(x)| \cdot\left|\Pi \varphi_{i}\left(x_{i}\right)\right| \leqq \Pi\left\|\varphi_{i}\right\| \cdot \sum_{x \in \Gamma}|F(x)| \cdot \Pi\left\|x_{i}\right\| ;
$$

on peut donc définir par $F \rightarrow \sum_{x \in T^{\prime}} F(x) . \Pi \varphi_{i}\left(x_{i}\right)$ une forme linéaire continue sur $L^{1}(T, v)$, de norme $\leqq I\left\|\varphi_{i}\right\| ;$ d'où, par passage au quotient, une forme linéaire continue, notée $\hat{\otimes} \varphi_{i}$, sur $\hat{\otimes} E_{i}$, caractérisée par

$$
\left(\hat{\otimes} \varphi_{i}\right)\left(\otimes x_{i}\right)=\Pi \varphi_{i}\left(x_{i}\right) ;
$$

et il est facile de voir que $\left\|\hat{\otimes} \varphi_{i}\right\|=\Pi\left\|\varphi_{i}\right\|$.

\section{\$ 3. Produits tensoriels infinis d'algèbres de Banach unitaires}

Soit $\left(A_{i}\right)_{i} \in_{I}$ une famille d'algèbres de Banach, chaque $A_{i}$ admettant un élément unité $e_{i}$; définissons $\Gamma$ et $v$ comme au $\S 2$ en prenant $\xi_{i}=e_{i}$; pour $F$ et $G \in L^{1}(\Gamma, v)$ posons

$$
(F G)(x)=\sum F(y) G(z)
$$

où la somme est étendue aux couples $y \in T, z \in \Gamma$ vérifiant $y_{i} z_{i}=x_{i}$ pour tout $i$; nous obtenons ainsi un élément $F G$ de $L^{1}(T, v)$ vérifiant $\|F G\| \leqq\|F\| \cdot\|G\| ; L^{1}(T, v)$ devient ainsi une algèbre de Banach; il est immédiat que $\bar{M}$ est un idéal bilatère.

Définition. On notera $\hat{\bigotimes}_{i \in I} A_{i}$ ou $\hat{\otimes} A_{i}$ l'algèbre de Banach $L^{1}(\Gamma, \nu) \mid \bar{M} ;$ la multiplication y est caractérisée par

$$
\left(\otimes x_{i}\right)\left(\otimes y_{i}\right)=\otimes x_{i} y_{i} \quad \forall\left(x_{i}\right),\left(y_{i}\right) \in \Gamma .
$$

L'algèbre $\widehat{\otimes} A_{i}$ et les morphismes canoniques $\varphi_{i}: A_{i} \rightarrow \widehat{\otimes} A_{i}$ jouissent de la propriété universelle suivante: étant donnée une algèbre de Banach unitaire $B$, en associant à tout morphisme continu unitaire $u: \hat{\otimes} A_{i} \rightarrow B$ la famille $\left(u \cdot \varphi_{i}\right)$, on obtient une bijection de l'ensemble des morphismes continus unitaires $\hat{\otimes} A_{i} \rightarrow B$ sur l'ensemble des familles de morphismes continus unitaires $u_{i}: A_{i} \rightarrow B$ deux à deux permutables. (c'est-à-dire dont les images sont deux à deux permutables). 
Supposons que les $A_{i}$ soient en outre involutives; on définit alors une involution sur $L^{\mathbf{1}}(\Gamma, v)$ en posant $F^{*}\left(\left(x_{i}\right)\right)=F\left(\left(x_{i}^{*}\right)\right) ; \bar{M}$ étant autoadjoint, il en résulte une involution sur $\otimes A_{i}$ caractérisée par

$$
\left(\otimes x_{i}\right)^{*}=\otimes x_{i}^{*} \quad \forall\left(x_{i}\right) \in \Gamma .
$$

Dans ce qui suit on note $C^{*}(A)$ la $C^{*}$-algèbre enveloppante d'une algèbre de Banach involutive unitaire $A$; pour la définition du produit tensoriel $\ddot{\otimes}$ d'une famille de $C^{*}$-algèbres, on renvoie à [3].

Proposition 4. Soit $\left(A_{i}\right)_{i} \in J$ une famille d'algèbres de Banach involutives unitaires; il existe un isomorphisme de $C^{*}\left(\hat{\otimes} A_{i}\right)$ sur $\check{\otimes} C^{*}\left(A_{i}\right)$ transformant, pour tout $\left(x_{i}\right) \in \Gamma$, l'image canonique de $\otimes x_{i}$ dans $C^{*}\left(\hat{\otimes} A_{i}\right)$ en $\otimes y_{i}$ où $y_{i}$ est l'image canonique de $x_{i}$ dans $C^{*}\left(A_{i}\right)$.

En effet les morphismes composés $A_{i} \rightarrow C^{*}\left(A_{i}\right) \rightarrow \check{\otimes} C^{*}\left(A_{i}\right)$ sont deux à deux permutables et par suite définissent un morphisme $\widehat{\otimes} A_{i} \rightarrow \check{\otimes} C^{*}\left(A_{i}\right)$, qui entraîne à son tour un morphisme $u: C^{*}\left(\hat{\otimes} A_{i}\right) \rightarrow \check{\otimes} C^{*}\left(A_{i}\right)$. D'autre part les morphismes composés $A_{i} \rightarrow \widehat{\otimes} A_{i} \rightarrow C^{*}\left(\hat{\otimes} A_{i}\right)$ définissent des morphismes $C^{*}\left(A_{i}\right) \rightarrow C^{*}\left(\hat{\otimes} A_{i}\right)$ qui sont deux à deux permutables, d'où un morphisme $v: \check{\otimes} C^{*}\left(A_{i}\right) \rightarrow C^{*}\left(\widehat{\otimes} A_{i}\right)$; enfin il est facile de vérifier que $u$ et $v$ sont réciproques.

\section{$\$ 4$. Produits continus de nombres complexes}

On désigne par $I$ un espace topologique localement compact dont chaque composante connexe est ouverte et fermée, et a un compactifié d'Alexandrov connexe par arcs et simplement connexe; par $\mu$ une mesure positive sur $I$ telle que toute composante connexe compacte de $I$ ait une mesure entière, positive ou nulle. On notera $\mathscr{K}(I)+1$ l'ensemble des fonctions complexes continues sur $I$, égales à 1 en dehors d'un compact. Une fonction sur $I$ sera souvent notée $i \rightarrow \lambda_{i}$ ou encore $\left(\lambda_{i}\right)$.

Soit $\left(\lambda_{i}\right)$ une fonction appartenant à $\mathscr{K}(I)+1$; on définit le produit continu $\widehat{\prod_{i \in I}} \lambda_{i}$ de cette fonction de la façon suivante: si on a $\lambda_{i}=0$ pour au moins un $i \in I$, on pose $\widehat{\Pi} \lambda_{i}=0$; dans le cas contraire on définit d'abord $\log \lambda_{i}$ en choisissant, dans toute composante connexe non compacte de $I$, la détermination égale à 0 a l'infini, et dans toute composante connexe compacte une détermination quelconque; et on pose

$$
\bigcap_{i \in I} \lambda_{i}=\exp \left(\int \log \lambda_{i} \cdot \mathrm{d} \mu(i)\right)
$$

ce qui a un sens puisque $\left(\log \lambda_{i}\right) \in \mathscr{K}(I)$ et que d'autre part, si l'on change la détermination de $\log \lambda_{i}$ sur une composante connexe $C$ où $\lambda_{i}$ ne prend pas la valeur 1, $C$ est compacte et l'on ne change pas la valeur de $\exp \left(\int_{C} \log \lambda_{i} \cdot d \mu(i)\right)$.

Il est clair que si $I$ est fini et si $\mu$ a une masse 1 en chaque point, on retrouve la notion ordinaire de produit $\Pi \lambda_{i}$. 
Proposition 5. Le produit continu $\widehat{\Pi} \lambda_{i}$ jouit des propriétés suivantes:

(i) si $\lambda_{i}$ est réel pour tout $i, \widehat{I} \lambda_{i}$ est réel;

(ii) si $\lambda_{i}$ est strictement positif pour tout $i, \widehat{\Pi} \lambda_{i}$ l'est aussi;

(iii) si $0<\lambda_{i} \leqq \lambda_{i}^{\prime}$ pour tout $i$, on a $\overparen{\Pi} \lambda_{i} \leqq \widehat{\Pi} \lambda_{i}^{\prime}$;

(iv) $\left|\widehat{\Pi} \lambda_{i}\right|=\widehat{\Pi}\left|\lambda_{i}\right|$;

(v) $\overparen{\Pi} \bar{\lambda}_{i}=\overline{\Pi \Pi \lambda_{i}}$;

(vi) $\overparen{\Pi} \lambda_{i} \lambda_{i}^{\prime}=\widehat{\Pi} \lambda_{i} \cdot \overparen{\Pi} \lambda_{i}^{\prime}$;

(vii) si $I$ est réunion d'ouverts $\left(I_{a}\right)_{a} \in_{A}$ deux $\grave{a}$ deux disjoints, on a $\prod_{i \in I} \lambda_{i}=\prod_{a \in A}\left(\prod_{i \in I_{a}} \lambda_{i}\right)$ où $\prod_{i \in I_{a}} \lambda_{i}$ est égal à 1 pour presque tout $a$.

La démonstration est facile; indiquons à titre d'exemple celle de (i) : on peut supposer $\lambda_{i} \neq 0$ pour tout $i$; si $C$ est une composante connexe où $\lambda_{i}>0$, on peut choisir $\log \lambda_{i}$ réel, alors $\exp \left(\int_{C} \log \lambda_{i} \cdot d \mu(i)\right)$ est réel; si $C$ est une composante connexe où $\lambda_{i}<0$, on peut choisir $\log \lambda_{i}=$ $=\log \left|\lambda_{i}\right|+\sqrt{-1} \pi ; C$ est compacte et on a

$$
\exp \left(\int_{C} \log \lambda_{i} \cdot d \mu(i)\right)=\exp \left(\int_{C} \log \left|\lambda_{i}\right| \cdot d \mu(i)\right) \cdot \exp (\sqrt{-1} \pi \mu(C))
$$

où $\mu(C)$ est entier, d'où l'assertion.

Nous poserons aussi $\overparen{\Pi} \lambda_{i}=\exp \left(\int \log \lambda_{i} \cdot d \mu(i)\right)$ pour toute fonction $\left(\lambda_{i}\right)$ strictement positive telle que $\left(\log \lambda_{i}\right)$ soit intégrable.

\section{§. Définition des produits tensoriels continus d'espaces de Banach}

On désigne par $I$ un espace topologique localement compact, réunion dénombrable de compacts, dont chaque composante connexe est ouverte et fermée, et a un compactifié d'Alexandrov connexe par arcs et simplement connexe; par $\mu$ une mesure positive sur $I$ telle que toute composante connexe compacte ait une mesure entière, positive ou nulle.

Nous appellerons famille continue d'espaces de Banach sur $I$ (notion différente de celle de champ continu de [2]) tout triplet $\left(\left(E_{i}\right)_{i} \in{ }_{I},\left(\xi_{i}\right)_{i} \in I, \Gamma\right)$ où $\left(E_{i}\right)_{i \in I}$ est une famille d'espaces de Banach complexes, $\xi_{i}$ un vecteur normé de $E_{i}$, et $\Gamma$ une partie de $\prod_{i \in I} E_{i}$ soumise aux conditions suivantes:

(i) la famille $\xi=\left(\xi_{i}\right)$ appartient à $\Gamma$;

(ii) si $x=\left(x_{i}\right) \in \Gamma$ on a $x_{i}=\xi_{i}$ en dehors d'un compact;

(iii) si $x=\left(x_{i}\right) \in \Gamma$ la fonction $i \rightarrow\left\|x_{i}\right\|$ est continue (et appartient alors à $\mathscr{K}(I)+1)$;

(iv) si $x=\left(x_{i}\right) \in \Gamma$ et si $\lambda=\left(\lambda_{i}\right) \in \mathscr{K}(I)+1$, la famille $\lambda x=\left(\lambda_{i} x_{i}\right)$ appartient à $\Gamma$;

(v) pour tout $x=\left(x_{i}\right) \in \Gamma$ vérifiant $x_{i} \neq 0 \forall i$ et toute fonction semi-continue inférieurement $i \rightarrow \varepsilon_{i}>1$, il existe une famille $\left(\varphi_{i}\right)_{i} \in I$ où $\varphi_{i}$ est une forme linéaire continue sur $E_{i}$, vérifiant les conditions suivantes: 
a) $\varphi_{i}\left(x_{i}\right)=\left\|x_{i}\right\|$ pour tout $i$

b) pour tout $y=\left(y_{i}\right) \in \Gamma$ la fonction $i \rightarrow \varphi_{i}\left(y_{i}\right)$ est continue;

c) $\left\|\varphi_{i}\right\| \leqq \varepsilon_{i}$ pour tout $i$

d) la fonction $i \rightarrow \log \left\|\varphi_{i}\right\|$ (positive) est $\mu$-mesurable.

Exemple. Soit $E$ un espace de Banach; nous appellerons famille continue constante associée $\dot{a} E$ toute famille continue obtenue en prenant $E_{i}=E$, pour $\left(\xi_{i}\right)$ une application continue de $I$ dans $E$ vérifiant $\left\|\xi_{i}\right\|=1$ et par ailleurs arbitraire, et pour $\Gamma$ l'ensemble des applications continues $i \rightarrow x_{i}$ de $I$ dans $E$ vérifiant $x_{i}=\xi_{i}$ en dehors d'un compact. Les axiomes (i) à (iv) sont trivialement vérifiés; pour vérifier (v) notons $A_{i}$ l'ensemble des formes $\varphi \in E^{\prime}$ telles que $\varphi\left(x_{i}\right)=\left\|x_{i}\right\| ; A_{i}$ est convexe et fermé dans $E^{\prime}$ muni de la topologie forte; montrons que l'application $i \rightarrow A_{i}$ est semicontinue inférieurement, autrement dit que pour tout ouvert $\Omega \subset E^{\prime}$ l'ensemble des $i$ tels que $A_{i} \cap \Omega \neq \theta$ est ouvert; en effet soient $i_{0}$ tel que $A_{i_{0}} \cap \Omega \neq \theta$ et $\varphi_{0} \in A_{i_{0}} \cap \Omega$; soit $\eta>0$ tel que $\left\|\varphi-\varphi_{0}\right\|<\eta \Rightarrow \varphi \in \Omega$; il existe un voisinage $V$ de $i_{0}$ tel que

$$
i \in V \Rightarrow \varphi_{0}\left(x_{i}\right) \neq 0 \text { et }\left|\left\|x_{i}\right\| / \varphi_{0}\left(x_{i}\right)-1\right| \cdot\left\|\varphi_{0}\right\|<\eta
$$

si alors $i \in V$ on vérifie immédiatement que la forme $\varphi_{0} \cdot\left\|x_{i}\right\| / \varphi_{0}\left(x_{i}\right)$ appartient à $A_{i} \cap \Omega$, qui est donc non vide. D'après le lemme ci-dessous il existe une application continue $i \rightarrow \varphi_{i}$ de $I$ dans $E^{\prime}$ telle que $\varphi_{i} \in A_{i}$ et $\left\|\varphi_{i}\right\| \leqq \varepsilon_{i} ;$ elle vérifie évidemment les conditions a) à d).

Lemme 1. (cf. [5], lemme 7.1). Soient I un espace topologique paracompact, $F$ un espace de Banach, $i \rightarrow A_{i}$ une application semi-continue inférieurement de I dans l'ensemble des parties convexes fermées de $F ; i \rightarrow \varepsilon_{i}$ une fonction semi-continue inférieurement vérifiant $\varepsilon_{i}>\inf _{y \in A_{i}}\|y\|$. Alors $i l$ existe une application continue $i \rightarrow y_{i}$ de $I$ dans $F$ telle que $y_{i} \in A_{i}$ et $\left\|y_{i}\right\| \leqq \varepsilon_{i}$ pour tout $i$.

Dans le cas présent on a $\inf _{y \in A_{i}}\|y\|=1$ en vertu du théorème de HahnBanach.

Remarque 2. L'axiome (v) n'est pas conséquence des autres, comme le montre l'exemple suivant: $I=[-1,+1], E_{i}=\mathbb{C}, \xi_{i}=-1$ pour $i<0$ et +1 pour $i \geqq 0, \Gamma=$ ensemble des fonctions $i \rightarrow \lambda_{i}$ et $i \rightarrow \lambda_{i} \xi_{i}$ où $\left(\lambda_{i}\right) \in \mathscr{K}(I)$. D'ailleurs toute famille continue $\left(\left(E_{i}\right),\left(\xi_{i}\right), \Gamma\right)$ où les $E_{i}$ sont de dimension 1 est «équivalente» à une famille constante au sens suivant: soit $\left(\varphi_{i}\right)$ une famille de formes linéaires telle que $\varphi_{i}\left(\xi_{i}\right)=\left\|\varphi_{i}\right\|=1$ et que $\left(\varphi_{i}\left(x_{i}\right)\right)$ soit continue pour tout $\left(x_{i}\right) \in \Gamma$; pour tout $\left(x_{i}\right) \in \Gamma$ on peut écrire $x_{i}=\lambda_{i} \xi_{i}$ où $\left(\lambda_{i}\right) \in \mathscr{K}(I)+1$ puisque $\varphi_{i}\left(x_{i}\right)=\lambda_{i}$; et il est facile de voir que l'on obtient ainsi un isomorphisme de $\Gamma$ sur $\mathscr{K}(I)+1$.

\section{Construction du produit tensoriel continu}

Notons $\mathscr{K}(T)$ l'ensemble des fonctions complexes $F$ sur $\Gamma$ telles que $F(x)=0$ sauf pour un nombre fini de $x$; notons $v$ la mesure positive 19 Commun. math. Phys., Vol. 5 
atomique sur $\Gamma$ attribuant à tout point $x=\left(x_{i}\right)$ la masse $v(x)=\overparen{I}\left\|x_{i}\right\|$; $L^{1}(\Gamma, v)$ est le séparé-complété de $\mathscr{K}(\Gamma)$ pour la semi-norme

$$
\|F\|=\sum_{x \in \Gamma}|F(x)| \cdot v(x)
$$

on notera $\delta_{x}$ la fonction égale à 1 en $x$ et à 0 ailleurs. Soit $M$ le sousespace vectoriel de $\mathscr{K}(\Gamma)$ engendré par les éléments de la forme

a) $\delta_{\left(\lambda_{i} x_{i}\right)}-\widehat{\Pi} \lambda_{i} \cdot \delta_{\left(x_{i}\right)}$ où $\left(x_{i}\right) \in \Gamma$ et $\left(\lambda_{i}\right) \in \mathscr{K}(I)+1$;

b) $\delta_{\left(x_{i}\right)}+\delta_{\left(y_{i}\right)}-\delta_{\left(z_{i}\right)}$ où $\left(x_{i}\right),\left(y_{i}\right),\left(z_{i}\right) \in \Gamma, x_{i}=y_{i}=z_{i}$ sauf en un point isolé $i_{0}$ et $z_{i_{0}}=x_{i_{0}}+y_{i_{0}}$;

soit $\bar{M}$ l'adhérence de l'image canonique de $M$ dans $L^{1}(\Gamma, v)$.

Définition. Nous appellerons produit tensoriel continu de la famille continue $\left(\left(E_{i}\right),\left(\xi_{i}\right), \Gamma\right)$ et noterons $\hat{\bigotimes}_{i \in I}^{\left(\xi_{i}\right) \Gamma} E_{i}$ ou plus simplement $\hat{\otimes}^{\xi \Gamma} E_{i}$ ou même $\hat{\otimes} E_{i}$ l'espace de Banach quotient $L^{1}(\Gamma, v) \mid \bar{M}$.

Si $I$ est discret, si $\mu$ a la masse +1 en chaque point et si on prend pour $\Gamma$ l'ensemble des $\left(x_{i}\right)$ telles que $x_{i}=\xi_{i}$ sauf pour un nombre fini de $i$, on retrouve la notion de produit tensoriel infini exposée au $\S 2$.

On notera $\dot{F}$ l'image canonique dans $\widehat{\otimes} E_{i}$ d'un élément $F$ de $L^{1}(T, v)$ et $\otimes x_{i}$ celle d'un élément $\delta_{x}$ où $x=\left(x_{i}\right) \in \Gamma$; les éléments $\otimes x_{i}$ forment une partie totale de $\hat{\otimes} E_{i}$; on a évidemment $\otimes \lambda_{i} x_{i}=\overparen{\Pi} \lambda_{i} \cdot \otimes x_{i}$ pour $\left(\lambda_{i}\right) \in \mathscr{K}(I)+1$.

Donnons-nous pour tout $i \in I$ une forme linéaire continue $\varphi_{i}$ non nulle sur $E_{i}$ de façon que les conditions suivantes soient vérifiées:

a) pour tout $\left(x_{i}\right) \in \Gamma$ la fonction $i \rightarrow \varphi_{i}\left(x_{i}\right)$ appartient à $\mathscr{K}(I)+1$;

b) la fonction $i \rightarrow \log \left\|\varphi_{i}\right\|$ est $\mu$-intégrable;

pour tout $F \in L^{1}(\Gamma, v)$ la famille $\left(F(x) \cdot \overparen{\Pi} \varphi_{i}\left(x_{i}\right)\right)_{x \in \Gamma}$ est sommable puisque

$$
\begin{aligned}
\sum_{x \in \Gamma}|F(x)| \cdot\left|\widehat{\Pi} \varphi_{i}\left(x_{i}\right)\right| & \leqq \sum_{x \in \Gamma}|F(x)| \cdot \overparen{\Pi}\left\|\varphi_{i}\right\| \cdot\left\|x_{i}\right\| \\
& =\overparen{\Pi}\left\|\varphi_{i}\right\| \cdot\|F\| ;
\end{aligned}
$$

on peut donc définir une forme linéaire continue $\bar{\varphi}$ sur $L^{1}(\Gamma, v)$, de norme $\leqq \widehat{\Pi}\left\|\varphi_{i}\right\|$, en posant

$$
\bar{\varphi}(F)=\sum_{x \in \Gamma} F(x) \cdot \widehat{\Pi} \varphi_{i}\left(x_{i}\right)
$$

la forme $\bar{\varphi}$ est visiblement nulle sur $\bar{M}$ et définit par passage au quotient une forme linéaire continue, notée $\widehat{\otimes} \varphi_{i}$, sur $\hat{\otimes} E_{i}$; d'où

Proposition 6. Pour toute famille $\left(\varphi_{i}\right)$ de formes linéaires continues vérifiant les conditions a) et b) ci-dessus, il existe une forme linéaire continue unique $\hat{\otimes} \varphi_{i}$ sur $\hat{\otimes}^{\xi \Gamma} E_{i}$ telle que $\left(\hat{\otimes} \varphi_{i}\right)\left(\otimes x_{i}\right)=\Pi \Pi \varphi_{i}\left(x_{i}\right)$ pour tout $\left(x_{i}\right) \in \Gamma$; on $a\left\|\hat{\otimes} \varphi_{i}\right\| \leqq \widehat{\Pi}\left\|\varphi_{i}\right\|$.

Proposition 7. Pour tout $\left(x_{i}\right) \in \Gamma$ on $a\left\|\otimes x_{i}\right\|=\widehat{\Pi}\left\|x_{i}\right\|$.

L'assertion étant triviale si $x_{i}=0$ pour au moins un $i$, supposons désormais $x_{i} \neq 0$ pour tout $i$; on a évidemment $\left\|\otimes x_{i}\right\| \leqq\left\|\delta_{x}\right\|=\widehat{\Pi}\left\|x_{i}\right\|$; 
pour prouver l'inégalité inverse prenons $\varepsilon>1 ; I$ étant dénombrable à l'infini il existe une fonction semi-continue inférieurement $i \rightarrow \varepsilon_{i}>1$ telle que $\int \log \varepsilon_{i} \cdot d \mu(i) \leqq \log \varepsilon$; choisissons une famille $\left(\varphi_{i}\right)$ vérifiant les conditions de l'axiome $(v)$; alors la fonction $i \rightarrow\left\|\varphi_{i}\right\|$ est $\mu$-intégrable et on a

d'autre part on a

$$
\begin{aligned}
\left\|\hat{\otimes} \varphi_{i}\right\| & \leqq \widehat{I I}\left\|\varphi_{i}\right\|=\exp \left(\int \log \left\|\varphi_{i}\right\| \cdot d \mu(i)\right) \leqq \\
& \leqq \exp \left(\int \log \varepsilon_{i} \cdot d \mu(i)\right) \leqq \varepsilon
\end{aligned}
$$

$$
\widehat{\Pi}\left\|x_{i}\right\|=\widehat{I} \varphi_{i}\left(x_{i}\right)=\left(\hat{\otimes} \varphi_{i}\right)\left(\otimes x_{i}\right) \leqq \varepsilon\left\|\otimes x_{i}\right\|
$$

ce qui, vu l'arbitraire de $\varepsilon$, démontre notre assertion.

Corollaire 2. On $\mathrm{a} \otimes x_{i}=0$ si et seulement si $x_{i}=0$ pour au moins un $i$.

Remarque 3. Si tous les $E_{i}$ sont de dimension 1 il en est de même de $\widehat{\otimes}^{\xi \Gamma} E_{i}$; en effet, d'après la remarque 1 , on peut supposer la famille constante et associée à $\mathbb{C}$; si $\left(x_{i}\right)$ et $\left(y_{i}\right) \in \Gamma$ avec $\otimes x_{i} \neq 0$, on a $x_{i} \neq 0$ pour tout $i$ et $\otimes y_{i}=\widehat{I} y_{i} \mid x_{i} \cdot \otimes x_{i}$.

Remarque 4 . Soient $I^{0}$ une partie ouverte de $I$ vérifiant les conditions imposées à $I$ au début du $\S, \xi^{0}$ la restriction de la famille $\left(\xi_{i}\right)$ à $I^{0}, \Gamma^{0}$ l'ensemble des restrictions à $I^{0}$ des familles $\left(x_{i}\right) \in \Gamma$ telles que $x_{i}=\xi_{i}$ en dehors d'un compact contenu dans $I^{0}$; il est facile de voir que $\left(\left(E_{i}\right)_{i \in I^{0}}\right.$, $\left.\left(\xi_{i}\right)_{i \in I^{0}}, \Gamma^{0}\right)$ est une famille continue d'espaces de Banach et de construire une application linéaire continue $T$ de $\widehat{\bigotimes}_{i \in I^{0}}^{\xi^{0} \Gamma^{0}} E_{i}$ dans $\hat{\bigotimes}_{i \in I}^{\xi} E_{i}$ transformant tout élément $\otimes y_{i}\left(\left(y_{i}\right) \in \Gamma^{0}\right)$ en $\otimes x_{i}$ où $x_{i}=y_{i}$ pour $i \in I^{0}$ et $\xi_{i}$ pour $i \in I^{0}$; on ignore si cette application $T$ est isométrique. Supposons maintenant $I^{0}$ ouverte et fermée et posons $I^{1}=I-I^{0}$; supposons de plus que quelles que soient les familles $\left(x_{i}\right),\left(y_{i}\right) \in \Gamma$ il existe une famille $\left(z_{i}\right) \in \Gamma$ telle que $z_{i}=x_{i}$ pour $i \in I^{0}$ et $z_{i}=y_{i}$ pour $i \in I^{1} ; \Gamma^{0}$ est alors l'ensemble des restrictions à $I^{0}$ des familles $\left(x_{i}\right) \in \Gamma$; en outre $T$ est isométrique, comme on le verra à la proposition 8 .

\section{§. Associativité du produit tensoriel continu}

On pourrait espérer un théorème général du type suivant: soient $I$ et $J$ deux espaces localement compacts, $T$ une application continue de $I$ sur $J$, $\mu$ et $\nu$ des mesures positives sur $I$ et $J, \mu=\int \mu_{j} d v(j)$ une désintégration de $\mu$ relative à $T$ et $\nu$; alors $\hat{\bigotimes}_{i \in I} E_{i}=\hat{\bigotimes}\left(\hat{\bigotimes}_{i \in I} E_{i}\right)$. Mais un tel résultat semble impossible à cause de l'exemple suivant: $I=[0,1] \times[0,1]$, $J=[0,1], T=$ première projection, $\mu, \nu, \mu_{j}=$ mesures de Lebesgue; notons $(j, k)$ un élément quelconque de $I$; supposons que l'on ait un $19 *$ 
champ de vecteurs $\left(x_{j k}\right)$ tel que

$$
\left\|x_{j k}\right\|= \begin{cases}\exp \left(-(j+k)^{-\frac{1}{2}}\right) & \text { si } j \text { ou } k \neq 0 \\ 0 & \text { si } j=k=0 ;\end{cases}
$$

alors $\prod_{K \in[0,1]}\left\|x_{j k}\right\|$ est égal à $\exp (-2(\sqrt{j+1}-\sqrt{\grave{j}}))$ pour $j \neq 0$ et à 0 pour $j=0$, donc ne dépend pas continûment de $j$.

Nous allons établir deux résultats partiels d'associativité, l'un en supposant $J$ discret, et l'autre en supposant que $I$ est le produit de $J$ par un espace discret.

Proposition 8. Soit $\left(\left(E_{i}\right)_{i \in I},\left(\xi_{i}\right)_{i \in I}, \Gamma\right)$ une famille continue d'espaces de Banach, I étant réunion d'une famille $\left(I^{a}\right)_{a} \in A$ d'ouverts deux à deux disjoints; pour tout $a \in A$ on note $\xi^{a}$ la restriction à $I^{a}$ de la famille $\xi=\left(\xi_{i}\right)$ et, plus généralement, $x^{a}$ la restriction à $I^{a}$ d'une famille quelconque $x=\left(x_{i}\right) \in \Gamma$; $\Gamma^{a}$ l'ensemble des $x^{a}$ où $\left(x_{i}\right) \in \Gamma$; on suppose réalisée la condition suivante:

(C) pour toute famille $\left(y_{a}\right) \in \prod_{a \in A} \Gamma^{a}$ telle que $y_{a}=\xi^{a}$ pour presque tout a il existe $\left(x_{i}\right) \in \Gamma$ tel que $y_{a}=x^{a}$ pour tout $a$.

Alors on peut considérer les produits tensoriels continus $\hat{\otimes}_{i \in I^{a}}^{\xi^{a} \Gamma^{a}} E_{i}$ et $i l$ existe un isomorphisme isométrique unique de $\hat{\bigotimes}_{i \in I}^{\xi T} E_{i}$ sur $\hat{\bigotimes}_{a \in A}^{\left(\eta_{a}\right)}\left(\hat{\bigotimes}_{i \in I^{a}}^{\xi^{a} \Gamma^{a}} E_{i}\right)$ transformant, pour toute famille $\left(x_{i}\right) \in \Gamma, \bigotimes_{i \in I} x_{i}$ en $\bigotimes_{a \in A}\left(\bigotimes_{i \in I^{a}} x_{i}\right)$. (On a posé $\eta_{a}=\bigotimes_{j \in I^{a}} \xi_{i}$.)

Il est immédiat que pour tout $a \in A,\left(\left(E_{i}\right)_{i \in I^{a}},\left(\xi_{i}\right)_{i \in I^{a}}, \Gamma^{a}\right)$ est une famille continue d'espaces de Banach; soient $\nu^{a}$ la mesure sur $\Gamma^{a}$ définie de la même facon que la mesure $v$ sur $\Gamma ; M^{a}$ le sous-espace vectoriel de $\mathscr{K}\left(\Gamma^{a}\right)$ défini de la même façon que $M$ pour $\mathscr{K}(\Gamma)$; en associant à tout $\left(x_{i}\right) \in \Gamma$ la famille $\left(x^{a}\right) \in \Pi \Gamma^{a}$ on obtient une application bijective de $\Gamma$ sur le sous-ensemble de $\Pi \Gamma^{a}$ formé des familles $\left(y_{a}\right)$ telles que $y_{a}=\xi^{a}$ pour presque tout a; cette application transforme $v$ en la mesure produit des $v^{a}$ d'après la prop. 5 (vii); d'après la prop. 3 il existe un isomorphisme isométrique $U$ de $L^{1}(\Gamma, v)$ sur $\hat{\bigotimes}_{a \in A}^{\left(\delta_{\xi^{a}}\right)} L^{1}\left(\Gamma^{a}, v^{a}\right)$ transformant, pour tout $x \in \Gamma, \delta_{x}$ en $\otimes \delta_{x^{a}}$. On va maintenant appliquer la prop. 2 aux sousespaces $\bar{M}^{a} C L^{1}\left(\Gamma^{a}, \nu^{a}\right)$; pour cela on doit montrer que $U(\bar{M})$ est engendré par les éléments $\otimes F^{a}$ où $F^{a} \in L^{1}\left(\Gamma^{a}, \nu^{a}\right), F^{a}=\delta_{\xi a}$ pour presque tout a et $F^{a} \in \bar{M}^{a}$ pour au moins un a; pour montrer qu'un tel $\otimes F^{a}$ appartient à $U(\bar{M})$ on peut supposer que $F^{a} \in M^{a}$ pour un indice $a_{0}$ et que pour $a \neq a_{0}, F^{a}$ est de la forme $\delta_{x^{a}}$ où $x \in \Gamma$, et la verification est alors triviale; pour montrer que ces éléments $\otimes F^{a}$ engendrent $U(\bar{M})$, on vérifie aisément que les éléments $U\left(\delta_{\lambda_{x}}-\Pi \lambda_{i} \delta_{x}\right)$ et $U\left(\delta_{x}+\delta_{y}-\delta_{z}\right)$ sont des 
combinaisons de tels éléments $\otimes F^{a}$. La prop. 2 fournit alors un isomorphisme isométrique de $L^{1}(\Gamma, v) / \bar{M}$ sur $\left.\otimes^{\left(\eta_{a}\right)} L^{1}\left(\Gamma^{a}, v^{a}\right) / \bar{M}^{a}\right)$, qui n'est autre que l'isomorphisme annoncé.

Proposition 9. Soient $J$ et $\mu_{0}$ un espace topologique et une mesure vérifiant les conditions indiquées en tête $d u \S 5 ; K$ un espace discret dénombrable, $\mu_{1}$ la mesure sur $K$ ayant la masse 1 en chaque point $;=J \times K$ et $\mu=\mu_{0} \otimes \mu_{1}$ vérifient les conditions requises; soit $\left(\left(E_{i}\right),\left(\xi_{i}\right), \Gamma\right)$ une famille continue d'espaces de Banach sur $I$; posons $i=(j, k)$; pour tout $j \in J$ posons $\xi_{j}=\bigotimes_{k \in K} \xi_{j k} \in \hat{\bigotimes}_{k \in K} E_{j k}$. Soit $\Gamma^{\prime}$ le sous-ensemble de $\prod_{j \in J}\left(\hat{\bigotimes}_{k \in K} E_{j k}\right)$ formé des familles $j \rightarrow \bigotimes_{k \in K} x_{j k}$ où $x \in \Gamma$; alors $\left(\left(\widehat{\bigotimes}_{k \in K} E_{j k}\right),\left(\xi_{j}\right), \Gamma^{\prime}\right)$ est une famille continue d'espaces de Banach sur $J$ et il existe un isomorphisme isométrique unique de $\hat{\bigotimes}_{i \in I}^{\xi \Gamma} E_{i}$ sur $\hat{\bigotimes}_{j \in J}^{\left(\xi_{j}\right) \Gamma^{\prime}}\left(\hat{\bigotimes}_{k \in K} E_{j k_{k}}\right)$ transformant, pour tout $x \in \Gamma, \bigotimes_{i \in I} x_{i}$ en $\bigotimes_{j \in J}\left(\bigotimes_{k \in K} x_{j k}\right)$.

Posons $E_{j}=\widehat{\bigotimes}_{k \in K} E_{j k}$ et, pour tout $x \in \Gamma, x_{j}=\bigotimes_{k \in K} x_{j k}$. Démontrons d'abord que $\left(\left(E_{j}\right),\left(\xi_{j}\right), \Gamma^{\prime}\right)$ est une famille continue; soit $x \in \Gamma$; on a $x_{j k}=\xi_{j k}$ si $(j, k)$ n'appartient pas à un compact $J_{0} \times K_{0}$ où $J_{0}$ est compact et $K_{0}$ fini; d'où l'axiome (ii); puis $\left\|x_{j}\right\|=\prod_{k \in K_{0}}\left\|x_{j k}\right\|$, d'où (iii); soit $\left(\lambda_{j}\right) \in \mathscr{K}(J)+\mathbf{1}$; choisissons $k_{0} \in K$; on peut écrire $\lambda_{j} x_{j}=\bigotimes_{k \in K} y_{j k}$ où $y_{j k}$ est égal à $\lambda_{j} x_{j k_{0}}$ pour $k=k_{0}$ et à $x_{j k}$ pour $k \neq k_{0}$; on a $y \in \Gamma$, donc $\left(\lambda_{j} x_{j}\right) \in \Gamma^{\prime}$; d'où (iv). Pour vérifier l'axiome (v) donnons-nous une fonction semi-continue inférieurement $j \rightarrow \varepsilon_{j}>1$; soit $\left(\alpha_{k}\right)_{k \in K}$ une famille de nombres réels strictement positifs tels que $\sum \alpha_{k}=1$; en vertu de l'axiome (v) appliqué à la famille $\left(\left(E_{i}\right),\left(\xi_{i}\right), \Gamma\right)$ il existe une famille $\left(\varphi_{j k}\right), \varphi_{j k} \in E_{j k}^{\prime}$, vérifiant

a) $\varphi_{j k}\left(x_{j k}\right)=\left\|x_{j k}\right\|$ quels que soient $j$ et $k$;

b) pour tout $y \in \Gamma$, la fonction $(j, k) \rightarrow \varphi_{j k}\left(y_{j k}\right)$ est continue;

c) $\left\|\varphi_{j k}\right\| \leqq \varepsilon_{j}^{\alpha_{k}}$;

d) la fonction $(j, k) \rightarrow\left\|p_{j k}\right\|$ est mesurable;

posons $\varphi_{j}=\bigotimes_{k \in k} \varphi_{j k}$ (cf. remarque 1); on a $\varphi_{j}\left(x_{j}\right)=\prod_{k \in K} \varphi_{j k}\left(x_{j_{k}}\right)$ $=\prod_{k \in K}\left\|x_{j k}\right\|=\left\|x_{j}\right\|$; si $y \in T, y_{j k}=x_{j k}=\xi_{j k}$ si $(j, k)$ n'appartient pas à un compact $J_{1} \times K_{1}$; alors $\varphi_{j}\left(y_{j}\right)=\prod_{k \in K} \varphi_{j k}\left(y_{j k}\right)=\prod_{k \in K_{1}} \varphi_{j k}\left(y_{j k}\right)$ dépend continûment de $j$; puis $\left\|\varphi_{j}\right\|=\prod_{k \in K}\left\|\varphi_{j k}\right\| \leqq \prod_{k \in K} \varepsilon_{j}^{\alpha_{k}}=\varepsilon_{j}$; enfin la fonction $j \rightarrow\left\|\varphi_{j}\right\|$ est mesurable comme produit d'une suite de fonctions mesurables. 
On peut donc considérer $E^{\prime}=\widehat{\bigotimes}_{j \in J}^{\left(\xi_{j}\right) \Gamma^{\prime}} E_{j}=L^{1}\left(\Gamma^{\prime}, v^{\prime}\right) / \bar{M}^{\prime} ;$ posons $E=\bigotimes_{i \in I}^{\xi \Gamma} E_{i} ;$ pour tout $F \in L^{1}(\Gamma, v)$ on a

$$
\sum_{x \in \Gamma}|F(x)| \cdot\left\|\bigotimes_{j \in J} x_{j}\right\|=\sum_{x \in \Gamma}|F(x)| \cdot \prod_{j k}\left\|x_{j k}\right\|=\left\|F^{\top}\right\| ;
$$

on peut donc définir une application linéaire continue $\bar{\Phi}$ de norme $\leqq 1$ de $L^{1}(I, \nu)$ dans $E^{\prime}$ par

$$
\bar{\Phi}(F)=\sum_{x \in I^{\prime}} F(x) \cdot \bigotimes_{j \in J} x_{j}
$$

$\bar{\Phi}$ est nulle sur $M$ (vérification facile) et définit par passage au quotient une application linéaire continue de norme $\leqq 1: \Phi: E \rightarrow E^{\prime}$, telle que

$$
\Phi\left(\bigotimes_{i \in I} x_{i}\right)=\bigotimes_{j \in J} x_{j}=\bigotimes_{j \in J}\left(\bigotimes_{k \in K} x_{j k}\right) \quad \text { pour tout } x \in \Gamma .
$$

Pour construire une application $\Psi$ réciproque de $\Phi$, on doit d'abord prouver que $x \in \Gamma, y \in \Gamma, \bigotimes_{k} x_{j k}=\bigotimes_{k} y_{j k} \forall j$ impliquent $\bigotimes_{i} x_{i}=\bigotimes_{i} y_{i}$; ceci étant évident si $x_{i}=0$ pour au moins un $i$, supposons désormais $x_{i} \neq 0$ pour tout $i$; pour tout $j \in J$ on peut écrire $y_{j k}=\lambda_{j k} x_{j k}$ avec $\lambda_{j k} \in \mathbb{C}, \lambda_{j k}=1$ pour presque tout $k$ et $\prod_{k} \lambda_{j k}=1$; ou encore $y_{i}=\lambda_{i} x_{i}$; choisissant $\varphi_{i} \in E_{i}^{\prime}$ de façon que $\varphi_{i}\left(x_{i}\right)=\left\|x_{i}\right\|$ et que $i \rightarrow \varphi_{i}\left(y_{i}\right)$ soit continue, on voit que $i \rightarrow \lambda_{i}=\varphi_{i}\left(y_{i}\right) /\left\|x_{i}\right\|$ a ppartient à $\mathscr{K}(I)+1$; comme $\Pi \lambda_{i}=1$ ceci entraîne $\bigotimes_{i} x_{i}=\bigotimes_{i} y_{i}$.

Ceci établi on peut considérer l'application $\bar{\Psi}$ de $\Gamma^{\prime}$ dans $E$ telle que $\bar{\Psi}\left(\left(x_{j}\right)_{j \in J}\right)=\bigotimes_{i} x_{i}$ pour tout $x \in \Gamma$; puis la prolonger en une application $\overline{\bar{\Psi}}: L^{1}\left(\Gamma^{\prime}, \nu^{\prime}\right) \rightarrow E$ définie par

$$
\overline{\bar{\Psi}}(G)=\sum_{\left(x_{j}\right) \in \Gamma^{\prime}} G\left(\left(x_{j}\right)\right) \cdot \bar{\Psi}\left(\left(x_{j}\right)\right)
$$

et on voit que $\overline{\bar{\Psi}}$ est linéaire de norme $\leqq 1$; montrons que $\overline{\bar{\Psi}}$ est nulle sur $M^{\prime}$ : soient d'abord $\left(x_{j}\right) \in \Gamma^{\prime}$ et $\left(\lambda_{j}\right) \in \mathscr{K}(J)+1$; choisissons $k_{0} \in K$ et posons

$$
\lambda_{i}=\lambda_{j k}= \begin{cases}\lambda_{j} & \text { si } k=k_{0} \\ 1 & \text { sinon } ;\end{cases}
$$

alors $\overline{\bar{\Psi}}\left(\delta_{\left(\lambda_{j} x_{j}\right)}-\widehat{\Pi} \lambda_{j} \cdot \delta_{\left(x_{j}\right)}\right)=\otimes \lambda_{i} x_{i}-\widehat{\Pi} \lambda_{i} \cdot \otimes x_{i}=0$.

Soient maintenant $\left(x_{j}\right),\left(y_{j}\right),\left(z_{j}\right) \in \Gamma^{\prime}$ tels que $x_{j}=y_{j}=z_{j}$ sauf en un point isolé $j_{0}$ et que $z_{j_{0}}=x_{j_{0}}+y_{j_{0}}$; pour montrer que $\overline{\bar{\Psi}}\left(\delta_{\left(x_{j}\right)}+\delta_{\left(y_{j}\right)}\right.$ $\left.-\delta_{\left(z_{j}\right)}\right)$ est nul, distinguons trois cas:

1) $x_{j_{0}}=y_{j_{0}}=z_{j_{0}}=0$ : trivial;

2) un seul de ces trois éléments est nul, par exemple $x_{j_{0}}$ : on peut écrire $z_{j_{0} k}=\lambda_{k} y_{j_{0} k}$ où $\lambda_{k} \in \mathbb{C}, \lambda_{k}=1$ pour presque tout $k$ et $\Pi \lambda_{k}=1$; alors $\otimes z_{i}=\otimes y_{i}$, d'où l'assertion; 
3) ces trois éléments sont non nuls: alors il existe un indice $k_{0}$ et des scalaires $a_{k}, b_{k}, c_{k}$ vérifiant

$$
\left\{\begin{array}{l}
a_{k}=b_{k}=c_{k}=1 \quad \text { pour presque tout } k \\
\Pi a_{k}=\Pi b_{k}=\Pi c_{k}=1 \\
a_{k} x_{j_{0} k}=b_{k} y_{j_{0} k}=c_{k} z_{j_{0} k} \quad \text { pour tout } k \neq k_{0} \\
a_{k_{0}} x_{j_{0} k_{0}}+b_{k_{0}} y_{j_{0} k_{0}}=c_{k_{0}} z_{j_{0} k_{0}}
\end{array}\right.
$$

posant $x_{j_{0} k}^{\prime}=a_{k} x_{j_{0} k}$ et $x_{j k}^{\prime}=x_{j k}$ pour $j \neq j_{0}$ et les définitions analogues pour $y_{j k}^{\prime}$ et $z_{j k}^{\prime}$, on a

$$
\begin{aligned}
\overline{\bar{\Psi}}\left(\delta_{\left(x_{j}\right)}+\delta_{\left(y_{j}\right)}-\delta_{\left(z_{j}\right)}\right) & =\overline{\bar{\Psi}}\left(\delta_{\left(x_{j}^{\prime}\right)}+\delta_{\left(y_{j}^{\prime}\right)}-\delta_{\left(z_{j}^{\prime}\right)}\right) \\
& =\otimes x_{i}^{\prime}+\otimes y_{i}^{\prime}-\otimes z_{i}^{\prime}=0 .
\end{aligned}
$$

L'application $\overline{\bar{\Psi}}$, étant nulle sur $M^{\prime}$, définit une application linéaire de norme $\leqq 1: \Psi: E^{\prime} \rightarrow E$ telle que pour tout $x \in \Gamma$ :

$$
\Psi\left(\bigotimes_{j} x_{j}\right)=\bigotimes_{i} x_{i}
$$

enfin on constate aisément que $\Phi$ et $\Psi$ sont mutuellement réciproques.

Corollaire 3. Soient $J$ un ensemble et, pour tout $j \in J,\left(\left(E_{i j}\right),\left(\xi_{i j}\right), \Gamma_{j}\right)$ une famille continue d'espaces de Banach sur un espace $I$; soit $\Gamma^{\prime}$ le sousensemble de $\prod_{i \in I} \hat{\bigotimes}_{j \in J} E_{i j}$ formé des familles $i \rightarrow \bigotimes_{j \in J} x_{i j}$ où pour tout $j$ la famille $i \rightarrow x_{i j}$ appartient $\grave{a} \Gamma_{j}$; posons $\xi_{i}=\bigotimes_{j \in J} \xi_{i j}$; alors $\left(\left(\bigotimes_{j \in J} E_{i j}\right)_{i} \in I\right.$, $\left.\left(\xi_{i}\right)_{i \in I}, \Gamma^{\prime}\right)$ est une famille continue d'espaces de Banach et il existe un isomorphisme isométrique de $\hat{\bigotimes}_{i \in I}^{\xi \Gamma^{\prime}}\left(\hat{\bigotimes}_{j \in J} E_{i j}\right)$ sur $\hat{\bigotimes}_{j \in J}\left(\hat{\bigotimes}_{i \in I}^{\xi \Gamma_{j}} E_{i j}\right)$ trans. formant, pour toute famille $j \rightarrow\left(x_{i j}\right)_{i \in J} \in \Gamma_{j}$, l'élément $\bigotimes_{i \in I}\left(\bigotimes_{j \in J} x_{i j}\right)$ en $\bigotimes_{\in J}\left(\bigotimes_{i \in I} x_{i j}\right)$

Considérons la famille continue $\left(\left(E_{i j}\right),\left(\xi_{i j}\right), \Gamma\right)$ sur $I \times J$ où $\Gamma$ est l'ensemble des familles $\left(x_{i j}\right)_{i j}$ telles que pour tout $j$ la famille $i \rightarrow x_{i j}$ appartienne à $\Gamma_{j}$; les prop. 8 et 9 donnent respectivement des isomorphismes de $\hat{\bigotimes}_{i j} E_{i j}$ sur $\hat{\bigotimes}_{j}\left(\bigotimes_{i} E_{i j}\right)$ et sur $\hat{\bigotimes}_{i}\left(\hat{\bigotimes}_{j} E_{i j}\right)$, et il suffit de les composer.

\section{§ 7. Produits tensoriels continus d'applications linéaires continues}

On définit $I$ et $\mu$ comme au début du $\S 5$.

Proposition 10. Soient $\left(\left(E_{i}\right),\left(\xi_{i}\right), \Gamma\right)$ et $\left(\left(E_{i}^{\prime}\right),\left(\xi_{i}^{\prime}\right), \Gamma^{\prime}\right)$ deux farnilles continues d'espaces de Banach sur $I$; soit pour tout $i, T_{i}$ une application linéaire continue non nulle de $E_{i}$ dans $E_{i}^{\prime}$; on suppose que

a) pour tout $x=\left(x_{i}\right) \in \Gamma$ la famille $\left(T_{i} x_{i}\right)$ appartient $\grave{a} \Gamma^{\prime}$; 
b) la fonction $i \rightarrow \log \left\|T_{i}\right\|$ est $\mu$-intégrable (noter qu'elle est automatiquement semi-continue inférieurement si pour tout $i$ les éléments $x_{i}$ où $x \in \Gamma$ sont partout dense dans $E_{i}$ );

dans ces conditions il existe une application linéaire continue unique $\hat{\otimes} T_{i}$ de $\hat{\otimes}^{\xi \Gamma} E_{i}$ dans $\hat{\otimes}^{\xi^{\prime} \Gamma^{\prime}} E_{i}^{\prime}$ transformant $\otimes x_{i}$ en $\otimes T_{i} x_{i}$ pour tout $x \in \Gamma ; s a$ norme est au plus égale $\grave{a} \Pi\left\|T_{i}\right\|$; elle lui est égale si l'on suppose que:

c) pour toute fonction continue $i \rightarrow k_{i}<1$ il existe $\left(x_{i}\right) \in \Gamma$ tel que $\left\|x_{i}\right\|=1$ et $\left\|T_{i} x_{i}\right\| \geqq k_{i}\left\|T_{i}\right\|$.

Pour tout $x \in \Gamma$ on a

$$
\begin{aligned}
\left\|\otimes T_{i} x_{i}\right\| & =\widehat{\Pi}\left\|T_{i} x_{i}\right\| \leqq \exp \left(\int \log \left\|T_{i}\right\| \cdot d \mu(i)\right) \cdot \exp \left(\int \log \left\|x_{i}\right\| d \mu(j)\right. \\
& =\widehat{\Pi}\left\|T_{i}\right\| \cdot \widehat{\Pi I}\left\|x_{i}\right\| ;
\end{aligned}
$$

on peut donc définir une application $\bar{T}$ de $L^{1}(\Gamma, v)$ dans $\hat{\otimes} E_{i}^{\prime}$ par

$$
\bar{T}(F)=\sum_{x \in \Gamma} F(x) \cdot \otimes T_{i} x_{i}
$$

$\bar{T}$ est linéaire, continue et de norme $\leqq \widehat{\Pi}\left\|T_{i}\right\|$; elle est nulle sur $M$ (vérification immédiate), donc définit une application de $\hat{\otimes} E_{i}$ dans $\hat{\otimes} E_{i}^{\prime}$ ayant les propriétés annoncées.

CQFD

Si $T_{i}$ est nul pour au moins un $i$ on pose $\hat{\otimes} T_{i}=0$. Les propriétés suivantes sont faciles a établir:

(i) pour toute fonction $\left(\lambda_{i}\right) \in \mathscr{K}(I)+1$ on a $\hat{\otimes} \lambda_{i} T_{i}=\widehat{\Pi} \lambda_{i} \cdot \hat{\otimes} T_{i}$;

(ii) soient $\left(S_{i}\right)$ et $\left(R_{i}\right)$ deux autres familles d'applications linéaires continues $E_{i} \rightarrow E_{i}^{\prime}$ vérifiant a) et b); supposons que $T_{i}=S_{i}=R_{i}$ sauf en un point isolé $i_{0}$ et que $T_{i_{0}}=S_{i_{0}}+R_{i_{0}}$; alors $\hat{\otimes} T_{i}=\hat{\otimes} S_{i}+\hat{\otimes} R_{i}$.

(iii) on suppose que pour tout $i$ les éléments $x_{i}$ où $x \in \Gamma$ sont partout denses dans $E_{i}$; soient $\left(\left(E_{i}^{\prime \prime}\right),\left(\xi_{i}^{\prime \prime}\right), \Gamma^{\prime \prime}\right)$ une troisième famille continue d'espaces de Baanch et $S_{i}: E_{i}^{\prime} \rightarrow E_{i}^{\prime \prime}$ des applications linéaires continues vérifiant les conditions analogues à a) et b); alors les applications $S_{i} \circ T_{i}$ vérifient aussi ces conditions et on a $\hat{\otimes}\left(S_{i} \circ T_{i}\right)=\left(\hat{\otimes} S_{i}\right) \circ\left(\hat{\otimes} T_{i}\right)$.

(iv) si les $T_{i}$ sont des isomorphismes isométriques et si pour tout $x^{\prime} \in \Gamma^{\prime}$ on a $\left(T_{i}^{-1} x_{i}^{\prime}\right) \in \Gamma$, alors $\hat{\otimes} T_{i}$ est un isomorphisme isométrique.

Remarque 5. La condition c) de la prop. 10 n'est pas toujours réalisée, on peut même avoir $\left\|T_{i}\right\|=1$ pour tout $i$ et $\hat{\otimes} T_{i}=0$, même si $\left(\left(E_{i}\right)\right.$, $\left.\left(\xi_{i}\right), \Gamma\right)$ est une famille constante associée à un espace hilbertien $E$ et si $i \rightarrow T_{i}$ est une application continue de $I$ dans $\mathscr{L}(E)$ muni de la topologie de la norme, comme le montre l'exemple suivant dû à A. Douady : prenons $E=\mathbb{C}^{2}, I=$ boule unité de $\mathbb{C}^{2}=$ ensemble des $i=\left(i_{1}, i_{2}\right)$ tels que $|i|^{2}=\left|i_{1}\right|^{2}+\left|i_{2}\right|^{2} \leqq 1, \quad \Gamma=$ ensemble des applications continues de $I$ dans $E$; pour tout $i \in I$ notons $T_{i}^{\prime}$ l'opérateur dans $E$ de projection orthogonale sur la droite contenant $i$, et posons $T_{i}=(1-|i|) \cdot i d+|i| T_{i}^{\prime}$ on a $\left\|T_{i}\right\|=1$, mais on va voirque pour toute application continue $i \rightarrow x_{i}$ de $I$ dans $E$ on a $T_{i} x_{i}=0$ pour au moins un $i$, ce qui prouvera que $\otimes T_{i}=0$; supposons le contraire: alors $\left(i \mid x_{i}\right) \neq 0$ pour tout $i ; i \cdot\left|\left(i \mid x_{i}\right)\right| \mid$ $\left(i \mid x_{i}\right)$, pour $|i|=1$, ne dépend que de l'image canonique de $i$ dans $S^{2}$ 
et on en déduit une section continue de l'application canonique $S^{3} \rightarrow S^{2}$; or il n'existe pas de telle section.

La proposition suivante montre que, en un certain sens, les produits tensoriels continus "permutent" aux quotients.

Proposition 11. Soit $\left(\left(E_{i}\right),\left(\xi_{i}\right), \Gamma\right)$ une famille continue d'espaces de Banach sur $I$; pour tout $i$ soient $F_{i}$ un sous-espace vectoriel fermé de $E_{i}$ et $T_{i}$ l'application canonique $E_{i} \rightarrow E_{i} \mid F_{i}$; on suppose réalisées les conditions suivantes:

a) $\left\|T_{i} \xi_{i}\right\|=1$ pour tout $i$

b) pour tout $x=\left(x_{i}\right) \in \Gamma$, la fonction $i \rightarrow d\left(x_{i}, F_{i}\right)$ est continue $(d(x, F)$ désigne la distance d'un point $x$ à une partie $F)$;

c) pour tout $x=\left(x_{i}\right) \in \Gamma$ tel que $T_{i} x_{i} \neq 0$ pour tout $i$ et toute fonction semi-continue inférieurement $i \rightarrow \varepsilon_{i}>1$ il existe une famille $\left(\varphi_{i}\right)$ où $\varphi_{i}$ est une forme linéaire continue sur $E_{i}$, nulle sur $F_{i}$, telle que $\varphi_{i}\left(x_{i}\right)=d\left(x_{i}, F_{i}\right)$, $\left\|\varphi_{i}\right\| \leqq \varepsilon_{i}$, que $i \rightarrow \varphi_{i}\left(y_{i}\right)$ soit continue pour tout $y \in \Gamma$ et que $i \rightarrow\left\|\varphi_{i}\right\|$ soit mesurable;

d) pour tout $x \in \Gamma$ et tout $\varepsilon>0$ il existe $y \in \Gamma$ tel que $y_{i}-x_{i} \in F_{i}$ et $\widehat{\Pi}\left\|y_{i}\right\| \leqq \widehat{\Pi}\left\|T_{i} x_{i}\right\|+\varepsilon$.

Soit $\Gamma^{\prime}$ le sous-ensemble de $\Pi\left(E_{i} \mid F_{i}\right)$ formé des familles $\left(T_{i} x_{i}\right)$ où $\left(x_{i}\right) \in \Gamma$; soit $K$ le sous-espace vectoriel fermé de $\hat{\otimes}^{\xi \Gamma} E_{i}$ engendré par les éléments de la forme $\otimes x_{i}$ ò̀ $x_{i} \in F_{i}$ pour au moins un $i$ ou de la forme $\otimes x_{i}-\otimes y_{i}$ où $x_{i}-y_{i} \in F_{i}$ pour tout $i$. Alors l'application $\hat{\otimes} T_{i}$ (cf. prop. 10) de $\hat{\otimes}^{\xi \Gamma} E_{i}$ dans $\hat{\otimes}^{\left(T_{i} \xi_{i}\right) \Gamma^{\prime}}\left(E_{i} / F_{i}\right)$ passe au quotient en uni morphisme isométrique de $\left(\hat{\otimes}^{\xi{ }^{T}} E_{i}\right) / K$ sur $\hat{\otimes}^{\left(T_{i} \xi_{i}\right) \Gamma}\left(E_{i} / F_{i}\right)$.

Démontrons d'abord que $\left(\left(E_{i} \mid F_{i}\right),\left(T_{i} \xi_{i}\right), \Gamma^{\prime}\right)$ est une famille continue: les axiomes (i), (ii) et (iv) sont trivialement vérifiés; (iii) l'est à cause de la condition b) et (v) à cause de c). Ensuite on peut considérer $T=\hat{\otimes} T_{i}$ puisque les conditions a) et b) de la prop. 10 sont vérifiées; $T$ étant visiblement nulle sur $K$ passe au quotient en une application linéaire continue $S$ de norme $\leqq 1$ de $\left(\hat{\otimes} E_{i}\right) / K$ dans $\hat{\otimes}\left(E_{i} \mid F_{i}\right)$ qui transforme $\omega\left(\otimes x_{i}\right)$ et $\otimes T_{i} x_{i}$ pour tout $x \in \Gamma$ (on note $\omega$ l'application canonique $\left.\hat{\otimes} E_{i} \rightarrow\left(\hat{\otimes} E_{i}\right) / K\right)$. D'autre part pour $x=\left(x_{i}\right) \in \Gamma, \omega\left(\otimes x_{i}\right)$ ne dépend que de $\left(T_{i} x_{i}\right)$ puisque si $T_{i} x_{i}=T_{i} y_{i}$ pour tout $i, \otimes x_{i}-\otimes y_{i} \in K$; d'où une application $\bar{R}: \Gamma^{\prime} \rightarrow\left(\hat{\otimes} E_{i}\right) / K$ transformant toute famille $\left(T_{i} x_{i}\right)$ en $\omega\left(\otimes x_{i}\right)$. Soient maintenant $F$ un élément de $L^{1}\left(\Gamma^{\prime}, v^{\prime}\right)$ et $\varepsilon$ un nombre $>0$; pour tout $x^{\prime} \in \Gamma^{\prime}$ tel que $F\left(x^{\prime}\right) \neq 0$ choisissons $\varepsilon_{x^{\prime}}>0$ de façon que $\sum_{x^{\prime} \in \Gamma^{\prime}}\left|F^{\prime}\left(x^{\prime}\right)\right| \varepsilon_{x^{\prime}} \leqq \varepsilon$, puis en utilisant la condition d), $x \in \Gamma$ tel que $T_{i} x_{i}=x_{i}^{\prime}$ et $\mathbb{\Pi}\left\|x_{i}\right\| \leqq \widehat{\Pi}\left\|x_{i}^{\prime}\right\|+\varepsilon_{x^{\prime}}$; on a alors

$$
\begin{aligned}
\sum_{x^{\prime} \in I^{\prime}}\left|F\left(x^{\prime}\right)\right| \cdot\left\|\bar{R}\left(x^{\prime}\right)\right\| & =\sum_{x^{\prime} \in \Gamma^{\prime}}\left|F\left(x^{\prime}\right)\right| \cdot \| \omega\left(\otimes x_{i} \| \leqq\right. \\
& \leqq \sum{ }^{\prime}\left|\left\|\left(x^{\prime}\right) \mid \cdot \widehat{\Pi}\right\| x_{i} \| \leqq\right. \\
& \leqq \sum\left|F\left(x^{\prime}\right)\right| \cdot\left(\bar{I}\left\|x_{i}^{\prime}\right\|+\varepsilon_{x^{\prime}}\right) \leqq \\
& \leqq\|F\|+\varepsilon ;
\end{aligned}
$$


ceci prouve que $\sum_{x^{\prime} \in \Gamma^{\prime}} F\left(x^{\prime}\right) \bar{R}\left(x^{\prime}\right)$ appartient à $\left(\hat{\otimes} E_{i}\right) / K$ et a une norme $\leqq\|F\|$; ceci définit une application linéaire continue $\overline{\bar{R}}$ de norme $\leqq 1$ de $L^{1}\left(\Gamma^{\prime}, \nu^{\prime}\right)$ dans $\left(\hat{\otimes} E_{i}\right) / K$ :

$$
\bar{R}(F)=\sum_{x^{\prime} \in \Gamma^{\prime}} F\left(x^{\prime}\right) \bar{R}\left(x^{\prime}\right)=\sum_{x^{\prime} \in \Gamma^{\prime}} F\left(x^{\prime}\right) \omega\left(\otimes x_{i}\right)
$$

où $\left(x_{i}\right) \in \Gamma$ et $T_{i} x_{i}=x_{i}^{\prime} ; \overline{\bar{R}}$ est nulle sur $M^{\prime}$ donc définit une application linéaire continue $R$ de norme $\leqq 1$ de $\widehat{\otimes}\left(E_{i} \mid F_{i}\right)$ dans $\left(\hat{\otimes} E_{i}\right) / K$ qui transforme $\otimes T_{i} x_{i}$ en $\omega\left(\otimes x_{i}\right)$ pour tout $\left(x_{i}\right) \in \Gamma$. Il est alors immédiat que $S$ et $R$ sont mutuellement réciproques.

Exemple. Prenons pour $\left(\left(E_{i}\right),\left(\xi_{i}\right), \Gamma\right)$ une famille constante associée à un espace de Banach $E$ (cf. $\S 5$, exemple) et pour $F_{i}$ un sous-espace vectoriel fermé fixe $F$ de $E$ tel que l'image canonique $T \xi_{i}$ de $\xi_{i}$ dans $E / F$ soit de norme 1 pour tout $i$. Alors les conditions a) et b) de la prop. 11 sont trivialement vérifiées; c) se vérifie en posant $\varphi_{i}=\psi_{i} \circ T$ où $i \rightarrow \psi_{i}$ est une application continue de $I$ dans $(E / F)^{\prime}$ muni de la topologie forte vérifiant $\psi_{i}\left(T x_{i}\right)=\left\|T x_{i}\right\|$ et $\left\|\psi_{i}\right\| \leqq \varepsilon_{i}$ (cf. $\S 5$, exemple); vérifions d): soit $U$ l'ensemble ouvert relativement compact des $i$ pour lesquels $x_{i} \neq \xi_{i}$; soit $i \rightarrow \varepsilon_{i}$ une fonction semi-continue inférieurement strictement positive telle que $\left(\exp \left(\int \varepsilon_{i} \cdot d \mu(i)-1\right) \cdot \widehat{\Pi}\left\|T x_{i}\right\| \leqq \varepsilon ;\right.$ posons

$$
A_{i}=\left\{\begin{array}{lll}
\left\{y \in E: T y=T x_{i}\right\} & \text { pour } & i \in U \\
\left\{\xi_{i}\right\} & \text { pour } & i \in U ;
\end{array}\right.
$$

on voit facilement que l'application $i \rightarrow A_{i}$ est semi-continue inférierement; d'après le lemme 1 il existe une application continue $i \rightarrow y_{i}$ de $I$ dans $E$ telle que $y_{i} \in A_{i}$ et $\left\|y_{i}\right\| \leqq e^{\varepsilon_{i}}\left\|T x_{i}\right\|$ pour tout $i$; alors $\left(y_{i}\right) \in \Gamma$ et en outre

$$
\widehat{\Pi}\left\|y_{i}\right\|=\exp \left(\int \log \left\|y_{i}\right\| \cdot d \mu(i)\right) \leqq \exp \left(\int \varepsilon_{i} \cdot d \mu(i)\right) \cdot \widehat{\Pi}\left\|T x_{i}\right\| \leqq \widehat{\Pi}\left\|T x_{i}\right\|+\varepsilon .
$$

Le raisonnement fait pour établir d) prouve aussi que $\Gamma^{\prime}$ est l'ensemble des applications continues $i \rightarrow z_{i}$ de $I$ dans $E / F$ telles que $z_{i}=T \xi_{i}$ en dehors d'un compact, c'est-à-dire que $\left((E / F),\left(T \xi_{i}\right), \Gamma^{\prime}\right)$ est une famille constante.

\section{§ 8. Produits tensoriels continus d'espaces $\boldsymbol{L}^{1}$}

La proposition suivante généralise la prop. 3 au cas des produits continus:

Proposition 12. Définissons $I$ et $\mu$ comme au début $d u \S 5$; soient, pour tout $i, X_{i}$ un ensemble, $\sigma_{i}$ une mesure positive atomique sur $X_{i}, \omega_{i}$ un point de $X_{i}$ de masse 1 ; soit $X$ une partie de $\Pi X_{i}$ vérifiant:

a) $\omega=\left(\omega_{i}\right) \in X$

b) si $\chi=\left(\chi_{i}\right) \in X$, on a $\chi_{i}=\omega_{i}$ en dehors d'un compact et la fonction $i \rightarrow \sigma_{i}\left(\chi_{i}\right)$ appartient $\grave{a} \mathscr{K}(I)+1$; 
soit $\Gamma$ le sous-ensemble de $\Pi L^{1}\left(X_{i}, \sigma_{i}\right)$ formé des familles $\left(\lambda_{i} \delta_{\chi_{i}}\right)$ où $\left(\lambda_{i}\right) \in \mathscr{K}(I)+1$ et $\left(\chi_{i}\right) \in X$; soit $\sigma$ la mesure positive atomique sur $X$ définie par $\sigma(\chi)=\widehat{\Pi} \sigma_{i}\left(\chi_{i}\right)$; soit enfin $\xi_{i}=\delta_{\omega_{i}} \in L^{1}\left(X_{i}, \sigma_{i}\right)$. Alors $\left(\left(L^{1}\left(X_{i}, \sigma_{i}\right),\left(\xi_{i}\right), \Gamma\right)\right.$ est une famille continue et il existe un isomorphisme isométrique unique de $\hat{\otimes}^{\xi \Gamma} L^{1}\left(X_{i}, \sigma_{i}\right)$ sur $L^{1}(X, \sigma)$ transformant tout élément $\otimes \delta_{\chi_{i}}$ où $\chi=\left(\chi_{i}\right) \in X$ en $\delta_{\chi}$.

On posera $E_{i}=L^{1}\left(X_{i}, \sigma_{i}\right)$. Démontrons d'abord que $\left(\left(E_{i}\right),\left(\xi_{i}\right), \Gamma\right)$ est une famille continue: les axiomes (i) à (iv) sont trivialement vérifiés; pour (v) il suffit de prendre la forme $\varphi_{i}$ sur $E_{i}$ définie par la fonction constante $\left|\lambda_{i}\right| \mid \lambda_{i} \in L^{\infty}\left(X_{i}, \sigma_{i}\right)$, c'est-à-dire

$$
\varphi_{i}(f)=\left|\lambda_{i}\right| \mid \lambda_{i} \cdot \sum_{\chi_{i} \in x_{i}} f\left(\chi_{i}\right) \cdot \sigma_{i}\left(\chi_{i}\right) \text { pour } f \in E_{i} .
$$

L'unicité de l'isomorphisme résulte du fait que les éléments $\otimes \delta_{\chi_{i}}$ forment un sous-ensemble total de $\hat{\otimes} E_{i}$.

Pour prouver l'existence partons d'abord d'un élément $F$ de $L^{1}(\Gamma, v)$; on remarquera que la donnée de $\left(\lambda_{i} \delta_{\chi_{i}}\right)$ détermine entièrement les $\lambda_{i}$ et $\chi=\left(\chi_{i}\right) ;$ on a

$$
\sum_{\left(\lambda_{i} \delta_{\chi_{i}}\right) \in T}\left|F\left(\left(\lambda_{i} \delta_{\chi_{i}}\right)\right)\right| \cdot \widehat{I I}\left|\lambda_{i}\right| \cdot \sigma(\chi)==\sum\left|F\left(\left(\lambda_{i} \delta_{\chi_{i}}\right)\right)\right| \cdot v\left(\left(\lambda_{i} \delta_{\chi_{i}}\right)\right)=\|F\| ;
$$

on peut donc définir une application linéaire continue $\bar{S}$ de norme $\leqq 1$ de $L^{1}(\Gamma, v)$ dans $L^{1}(X, \sigma)$ par

$$
\bar{S}(F)=\sum_{\left(\lambda_{i} \delta_{\chi_{i}}\right) \in T} F\left(\left(\lambda_{i} \delta_{\chi_{i}}\right)\right) \cdot \overparen{I} \lambda_{i} \cdot \delta_{\chi}
$$

$\bar{S}$ est nulle sur $M$ (vérification facile), donc définit une application linéaire continue $S$ de norme $\leqq 1$ de $\hat{\otimes} E_{i}$ dans $L^{1}(X, \sigma)$ qui transforme tout élément $\otimes \delta_{\chi_{i}}$ en $\delta_{z}$.

Inversement pour tout élément $f$ de $L^{1}(X, \sigma)$ on a

$$
\sum_{\chi \in x}|f(\chi)| \cdot\left\|\otimes \delta_{z_{i}}\right\|=\sum_{\chi \in x}|f(\chi)| \cdot \widehat{\Pi} \sigma_{i}\left(\chi_{i}\right)=\|f\|
$$

et on peut définir une application linéaire continue $T$ de norme $\leqq 1$ de $L^{1}(X, \sigma)$ dans $\hat{\otimes} E_{i}$ par

$$
T(f)=\sum_{\chi \in x} f(\chi) \cdot \otimes \delta_{\chi_{i}}
$$

enfin il est immédiat que $S$ et $T$ sont mutuellement réciproques.

Exemple. On peut réaliser les conditions de la prop. 12 en prenant pour $X_{i}$ un ensemble fixé $X_{0}$ muni d'une topologie, pour $\sigma_{i}$ une mesure atomique fixée $\sigma_{0}$ sur $X_{0}$ telle que la fonction sur $X_{0}: \chi \rightarrow \sigma_{0}(\{\chi\})$ soit continue; pour $\omega_{i}$ un élément de $X_{0}$ de masse 1 et dépendant continûment de $i$; et pour $X$ l'ensemble des applications continues $i \rightarrow \chi_{i}$ de $I$ dans $X_{0}$ telles que $\chi_{i}=\omega_{i}$ en dehors d'un compact. 


\section{§ 9. Produit tensoriel inductif d'une famille continue constante d'espaces de Banach}

Définissons $I$ et $\mu$ comme au début du $\S 5$; considérons la famille $\left(\left(E_{i}\right),\left(\xi_{i}\right), \Gamma\right)$ continue constante associée à un espace de Banach $E$ et à une application continue $i \rightarrow \xi_{i}$ (cf. $\S 5$, exemple); notons $\Phi$ l'ensemble des applications continues $i \rightarrow \varphi_{i}$ de $I$ dans $E^{\prime}$ muni de la topologie forte telles que $\varphi_{i} \neq 0$ pour tout $i$, que $\varphi_{i}\left(\xi_{i}\right)=1$ en dehors d'un compact et que la fonction $i \rightarrow \log \left\|\varphi_{i}\right\|$ soit $\mu$-intégrable. Rappelons (§5) que l'on peut associer à toute $\left(\varphi_{i}\right) \in \Phi$ une forme linéaire continue $\bar{\varphi} \operatorname{sur} L^{1}(\Gamma, v)$ telle que

$$
\bar{\varphi}(F)=\sum_{\left(x_{i}\right) \in \Gamma} F\left(\left(x_{i}\right)\right) \cdot \Pi \varphi_{i}\left(x_{i}\right) .
$$

Définition. On notera $\hat{\hat{\otimes}}^{\xi T} E_{i}$ ou $\hat{\hat{\otimes}} E_{i}$ l'espace de Banach séparécomplété de $L^{1}(\Gamma, \nu)$ pour la semi-norme $q$ définie par

$$
q(F)=\sup _{\left(\varphi_{i}\right) \in \Phi} \mid \bar{\varphi}(F)\|\widehat{\Pi}\| \varphi_{i} \| .
$$

On a évidemment $q(F) \leqq\|F\|$; d'autre part $q$ est nulle sur $M$ et on peut aussi définir $\hat{\hat{\otimes}} E_{i}$ comme le séparé-complété de $\hat{\otimes} E_{i}$ pour la semi-norme $x \rightarrow \sup \left(\hat{\otimes} \varphi_{i}\right)(x) / \widehat{I}\left\|\varphi_{i}\right\|$. On notera encore $\otimes x_{i}$ l'image canonique $\left(\varphi_{i}\right) \in \Phi$

de $\delta_{\left(x_{i}\right)}$ dans $\hat{\hat{\otimes}} E_{i}$ pour toute famille $\left(x_{i}\right) \in \Gamma$; et on a $q\left(\otimes x_{i}\right)=\widehat{\Pi}\left\|x_{i}\right\|$ d'après la démonstration de la prop. 7 .

Proposition 13. Si F est un sous-espace de Banach de E contenant tous les $\xi_{i}$, le produit tensoriel $\hat{\hat{\otimes}} F_{i}$ de la famille constante correspondant $\grave{a} F$ et $\dot{a} i \rightarrow \xi_{i}$ s'identifie isométriquement $\dot{a}$ un sous-espace de Banach de $\hat{\otimes} E_{i}$.

Définissons $\Delta, \sigma$ et $\Psi$ à partir de $F$ comme $\Gamma, v$ et $\Phi$ sont définis à partir de $E ; \Delta$ est un sous-ensemble de $\Gamma$ et $\sigma$ est la restriction de $v$ à $\Delta$; $\widehat{\hat{\otimes}} F_{i}$ est le séparé-complété de $L^{1}(\Delta, \sigma)$, sous-espace de $L^{1}(T, v)$, pour la semi-norme $r(F)=\sup _{(\psi) \in \Psi}|\bar{\psi}(F)| \mid \Gamma\left\|\psi_{i}\right\|$; il suffit donc de prouver que $q(F)=r(F)$ pour toute $F \in L^{1}(\Delta, \sigma)$. On a évidemment $q(F) \leqq r(F)$; pour établir l'inégalité inverse prenons $\varepsilon>0$ et $\left(\psi_{i}\right) \in \Psi$ telle que $\mid \bar{\psi}(F)\|\widehat{I}\| \psi_{i} \| \geqq r(F) e^{-\varepsilon / 2} ;$ soit $i \rightarrow \varepsilon_{i}>0$ une fonction semi-continue inférieurement telle que $\int \varepsilon_{i} d \mu(i) \leqq \varepsilon / 2$; en vertu du lemme 1 il existe une application continue $i \rightarrow \varphi_{i}$ de $I$ dans $E^{\prime}$ telle que $\varphi_{i} \mid F=\psi_{i}$ et $\left\|\varphi_{i}\right\| \leqq e^{\varepsilon_{i}}\left\|\psi_{i}\right\|$ pour tout $i$; alors $\left(\varphi_{i}\right) \in \Phi$ et on a

$$
\begin{aligned}
q(F) & \geqq|\bar{\varphi}(F)|\left|\widehat{\Pi}\left\|\varphi_{i}\right\|=\right| \bar{\psi}(F) \mid \widehat{\Pi}\left\|\varphi_{i}\right\| \geqq \\
& \geqq|\bar{\psi}(F)| \mid e^{\varepsilon / 2} \widehat{\Pi}\left\|\psi_{i}\right\| \geqq r(F) e^{-\varepsilon}
\end{aligned}
$$

d'où $q(F) \geqq r(F)$. 


\section{§10. Produits tensoriels continus d'algèbres de Banach unitaires}

Nous appellerons famille continue d'algèbres de Banach toute famille continue $\left(\left(A_{i}\right)_{i \in I},\left(e_{i}\right)_{i \in I}, \Gamma\right)$ où les $A_{i}$ sont des algèbres de Banach, $e_{i}$ l'élément unité de $A_{i}$, et où $\left(x_{i}\right) \in \Gamma$ et $\left(y_{i}\right) \in \Gamma$ impliquent $\left(x_{i} y_{i}\right) \in \Gamma$.

Proposition 14. Si $\left(\left(A_{i}\right),\left(e_{i}\right), T\right)$ est une famille continue d'algèbres de Banach il existe sur $\hat{\otimes} A_{i}$ une structure d'algèbre de Banach et une seule telle que $\otimes x_{i} \cdot \otimes y_{i}=\otimes x_{i} y_{i}$ pour $\left(x_{i}\right)$ et $\left(y_{i}\right) \in \Gamma$. Si les $A_{i}$ sont involutives et si $\left(x_{i}^{*}\right) \in \Gamma$ dès que $\left(x_{i}\right) \in \Gamma$, il existe sur $\hat{\otimes} A_{i}$ une involution et une seule telle que $\left(\otimes x_{i}\right)^{*}=\otimes x_{i}^{*}$.

D'abord $\Gamma$ est un monoïde semi-normé pour l'opération $x y=\left(x_{i} y_{i}\right)$ et la semi-norme $\nu$; par un procédé standard on peut munir $L^{1}(\Gamma, v)$ d'une structure d'algèbre de Banach en posant $(F G)(x)=\sum_{y=x} F(y) G(z)$; on a alors $\delta_{x} \delta_{y}=\delta_{x y}$. On va voir que $\bar{M}$ est un idéal bilatère de cette algèbre; pour cela il suffit de montrer que, avec les notations du $\S 5$,

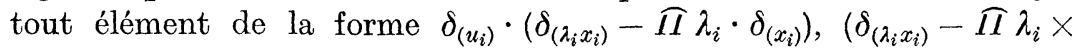
$\left.\times \delta_{\left(x_{i}\right)}\right) \cdot \delta_{\left(u_{i}\right)}, \delta_{u} \cdot\left(\delta_{x}+\delta_{y}-\delta_{z}\right),\left(\delta_{x}+\delta_{y}-\delta_{z}\right) \cdot \delta_{u}$ appartient à $M$, ce qui ne présente aucune difficulté. La structure d'algèbre cherchée sur $\hat{\otimes} A_{i}$ est alors la structure quotient; son unicité résulte de la totalité des éléments $\otimes x_{i}$. Supposons maintenant les $A_{i}$ involutives et $\left(x_{i}^{*}\right) \in \Gamma$ dès que $\left(x_{i}\right) \in \Gamma ; L^{1}(\Gamma, v)$ est une algèbre de Banach involutive si l'on pose $F^{*}\left(\left(x_{i}\right)\right)=\overline{F\left(\left(x_{i}^{*}\right)\right)}$; et l'involution passe au quotient puisque, comme on le voit immédiatement, l'idéal $\bar{M}$ est autoadjoint.

Proposition 15. Soient $\left(\left(A_{i}\right),\left(e_{i}\right), \Gamma\right)$ une famille continue d'algèbres de Banach, $\left(\left(E_{i}\right),\left(\xi_{i}\right), \Delta\right)$ une famille continue d'espaces de Banach, et, pour tout $i, \pi_{i}$ une représentation continue de $A_{i}$ dans $E_{i}$; on suppose que

a) pour tout $i$ les éléments $x_{i}$ où $\left(x_{i}\right) \in \Delta$ sont partout denses dans $E_{i}$;

b) la fonction $i \rightarrow \log \left\|\pi_{i}\right\|$ est $\mu$-intégrable;

c) pour tout $\left(a_{i}\right) \in \Gamma$ et tout $\left(x_{i}\right) \in \Delta$ on $a\left(\pi_{i}\left(a_{i}\right) \cdot x_{i}\right) \in \Delta$;

dans ces conditions il existe une représentation continue unique $\hat{\otimes} \pi_{i}$ de $\hat{\otimes} A_{i}$ dans $\hat{\otimes} E_{i}$ telle que $\left(\hat{\otimes} \pi_{i}\right)\left(\otimes a_{i}\right)\left(\otimes x_{i}\right)=\otimes\left(\pi_{i}\left(a_{i}\right) \cdot x_{i}\right)$ pour $\left(a_{i}\right) \in \Gamma$ et $\left(x_{i}\right) \in \Delta ;$ sa norme est au plus égale à $\widehat{\Pi}\left\|\pi_{i}\right\|$.

Pour tout $\left(a_{i}\right) \in \Gamma$ la famille des opérateurs $\pi_{i}\left(a_{i}\right)$ vérifie les hypothèses de la prop. 10 et on a même $\log \left\|\pi_{i}\left(a_{i}\right)\right\| \leqq \log \left\|\pi_{i}\right\|+\log \left\|a_{i}\right\|$; on peut donc considérer $\hat{\otimes} \pi_{i}\left(a_{i}\right) \in \mathscr{L}\left(\hat{\otimes} E_{i}\right)$, opérateur de norme au plus égale à $\Pi\left\|\pi_{i}\right\| \cdot \Pi\left\|a_{i}\right\| ;$ pour tout $F^{\prime} \in L^{1}(\Gamma, v)$ on a

$$
\sum_{a \in \Gamma}|F(a)| \cdot\left\|\hat{\otimes} \pi_{i}\left(a_{i}\right)\right\| \leqq \widehat{I}\left\|\pi_{i}\right\| \cdot\|F\|
$$

ce qui permet de définir une application linéaire continue $\bar{\pi}$ de norme $\leqq \widehat{\Pi}\left\|\pi_{i}\right\|$ de $L^{1}(T, v)$ dans $\mathscr{L}\left(\hat{\otimes} E_{i}\right)$ par $\bar{\pi}(F)=\sum_{a \in \Gamma} F(a) \cdot \hat{\otimes} \pi_{i}\left(a_{i}\right) ;$ 
$\bar{\pi}$ est un morphisme d'algèbres: en effet pour $F$ et $G \in L^{1}(\Gamma, v)$ on a

$$
\begin{gathered}
\bar{\pi}(F G)=\sum_{a \in \Gamma} \sum_{b c=a} F(b) G(c) \cdot \hat{\otimes} \pi_{i}\left(b_{i}\right) \pi_{i}\left(c_{i}\right) \\
\hat{\otimes} \pi_{i}\left(b_{i}\right) \pi_{i}\left(c_{i}\right)=\hat{\otimes} \pi_{i}\left(b_{i}\right) \cdot \hat{\otimes} \pi_{i}\left(c_{i}\right)
\end{gathered}
$$

d'après la propriété (iii) du $\S 7$; donc

$$
\bar{\pi}(F G)=\sum_{b, c} F(b) \cdot \hat{\otimes} \pi_{i}\left(b_{i}\right) \cdot G(c) \cdot \hat{\otimes} \pi_{i}\left(c_{\imath}\right)=\bar{\pi}(F) \bar{\pi}(G) ;
$$

$\bar{\pi}$ est nulle sur les éléments $\delta_{\left(\lambda_{i} a_{i}\right)}-\widehat{I} \lambda_{i} \delta_{\left(a_{i}\right)}$ et $\delta_{a}+\delta_{b}-\delta_{c}$ en vertu des propriétés (i) et (ii) du $\S 7$, donc nulle sur $\bar{M}$; d'où le résultat.

\section{Relation entre produits tensoriels continus et produits croisés}

Rappelons la définition des produits croisés (cf. [7]): soient $A$ une algèbre de Banach, d'unité $e$, et $G$ un groupe, d'élément neutre $\varepsilon$, opérant dans $A$ par automorphismes isométriques notés $x \rightarrow s \cdot x(x \in A, s \in G)$; le produit croisé de $A$ par $G$ est l'algèbre de Banach $L^{1}(G, A)$ des applications $F$ de $G$ dans $A$, intégrables pour la mesure attribuant la masse 1 à chaque point de $G$, munie de la multiplication suivante:

$$
\left(F F^{\prime}\right)(s)=\sum_{t \in G} F(t) t \cdot F^{\prime}\left(t^{-1} s\right) ;
$$

on notera $U$ et $V$ les applications de $G$ et de $A$ dans $L^{1}(G, A)$ définies respectivement par

$$
\begin{aligned}
& (U(s))(t)=\left\{\begin{array}{l}
e \text { si } t=s \\
0 \text { sinon }
\end{array}\right. \\
& (V(x))(t)=\left\{\begin{array}{l}
x \text { si } t=\varepsilon \\
0 \text { sinon } ;
\end{array}\right.
\end{aligned}
$$

les éléments $U(s)$ et $V(x)$ engendrent $L^{1}(G, A)$ puisque tout élément $F s^{\prime}$ écrit $F=\sum_{s \in G} V(F(s)) U(s)$; notons aussi l'identité fondamentale

$$
U(s) V(x)=V(s \cdot x) U(s) .
$$

Soient maintenant $\left(\left(A_{i}\right),\left(e_{i}\right), \Gamma\right)$ une famille continue d'algèbres de Banach et $A$ son produit tensoriel; pour tout $i$ soit $G_{i}$ un groupe d'élément neutre $\varepsilon_{i}$, opérant dans $A_{i}$ par automorphismes isométriques; notons $U_{i}$ et $V_{i}$ les applications correspondantes de $G_{i}$ et $A_{i}$ dans $L^{1}\left(G_{i}, A_{i}\right)$; soit $G$ le sous-ensemble de $\Pi I G_{i}$ formé des familles $s=\left(s_{i}\right)$ telles que $s_{i}$ $=\varepsilon_{i}$ en dehors d'un compact et que l'on ait $\left(s_{i} \cdot x_{i}\right)$ et $\left(s_{i}^{-1} \cdot x_{i}\right) \in \Gamma$ dès que $\left(x_{i}\right) \in \Gamma ; G$ est un sous-groupe de $\Pi G_{i}$ et opère dans $A$ par automorphismes isométriques tels que $s \cdot \otimes x_{i}=\otimes\left(s_{i} \cdot x_{i}\right)$ (cf. prop. 10 et propriétés suivantes). Soit $\Theta$ le sous-ensemble de $\Pi L^{1}\left(G_{i}, A_{i}\right)$ formé des familles $i \rightarrow V_{i}\left(x_{i}\right) U_{i}\left(s_{i}\right)$ où $\left(s_{i}\right) \in G$ et $\left(x_{i}\right) \in \Gamma$; on notera $1_{i}$ l'élément unité $U_{i}\left(\varepsilon_{i}\right)=V_{i}\left(e_{i}\right)$ de $L^{1}\left(G_{i}, A_{i}\right)$. 
Proposition 16. La famille d'algèbres de Banach $\left(\left(L^{1}\left(G_{i}, A_{i}\right)\right),\left(1_{i}\right), \Theta\right)$ est continue et il existe un isomorphisme d'algèbres de Banach unique de $L^{1}(G, A)$ sur $\hat{\otimes}^{\left(1_{i}\right) \Theta} L^{1}\left(G_{i}, A_{i}\right)$ transformant $U(s)$ en $\otimes U_{i}\left(s_{i}\right)$ pour tout $s=\left(s_{i}\right) \in G$ et $V\left(\otimes x_{i}\right)$ en $\otimes V_{i}\left(x_{i}\right)$ pour tout $\left(x_{i}\right) \in \Gamma$.

Soit $\Delta$ le sous-ensemble de $\Pi L^{1}\left(G_{i}\right)$ formé des familles $\left(\lambda_{i} \delta_{s_{i}}\right)$ où $\left(\lambda_{i}\right) \in \mathscr{K}(I)+1$ et $\left(s_{i}\right) \in G$; d'après la prop. $12,\left(\left(L^{1}\left(G_{i}\right), \delta_{\varepsilon_{i}}\right), \Delta\right)$ est une famille continue. On sait qu'il existe pour tout $i$ un isomorphisme isométrique d'espaces de Banach de $L^{1}\left(G_{i}, A_{i}\right)$ sur $L^{1}\left(G_{i}\right) \hat{\otimes} A_{i}$ transformant tout élément $V_{i}\left(x_{i}\right) U_{i}\left(s_{i}\right)$ en $\delta_{s_{i}} \otimes x_{i}$; on peut donc identifier $\left(\left(L^{1}\left(G_{i}, A_{i}\right)\right.\right.$, $\left.\left(\mathrm{l}_{i}\right), \Theta\right)$ à $\left(\left(L^{1}\left(G_{i}\right) \hat{\otimes} A_{i},\left(\delta_{\varepsilon_{i}} \hat{\otimes} e_{i}\right), \Gamma^{\prime}\right)\right.$ où $\Gamma^{\prime}$ est le sous-ensemble de $\Pi\left(L^{1}\left(G_{i}\right) \hat{\otimes} A_{i}\right)$ formé des familles $\left(\delta_{s_{i}} \otimes x_{i}\right)$; le corollaire 3 montre alors que ces familles sont continues, puis qu'il existe un isomorphisme isométrique de $\hat{\otimes} L^{1}\left(G_{i}, A_{i}\right) \operatorname{sur} \hat{\otimes} L^{1}\left(G_{i}\right) \hat{\otimes} A$ transformant $\otimes V_{i}\left(x_{i}\right) U_{i}\left(s_{i}\right)$ en $\left(\otimes \delta_{s_{i}}\right) \otimes\left(\otimes x_{i}\right)$; d'après la prop. 12 il existe un isomorphisme isométrique de $\hat{\otimes} L^{1}\left(G_{i}\right)$ sur $L^{1}(G)$ transformant $\otimes \delta_{s_{i}}$ en $\delta_{s}$; enfin on a un isomorphisme isométrique de $L^{1}(G) \hat{\otimes} A$ sur $L^{1}(G, A)$ transformant $\delta_{s} \otimes$ $\left(\otimes x_{i}\right)$ en $V\left(\otimes x_{i}\right) U(s)$; en composant ces isomorphismes on obtient un isomorphisme isométrique d'espaces de Banach de $\hat{\otimes} L^{1}\left(G_{i}, A_{i}\right)$ sur $L^{1}(G, A)$ transformant $\otimes V_{i}\left(x_{i}\right) U_{i}\left(s_{i}\right)$ en $V\left(\otimes x_{i}\right) U(s)$; pour montrer que cet isomorphisme respecte les produits il suffit de montrer qu'il transforme $\otimes V_{i}\left(x_{i}\right) U_{i}\left(s_{i}\right) \cdot \otimes V_{i}\left(x_{i}^{\prime}\right) U_{i}\left(s_{i}^{\prime}\right)$ en $V\left(\otimes x_{i}\right) U(s) V\left(\otimes x_{i}^{\prime}\right) U\left(s^{\prime}\right)$, ce qui est facile en tenant compte de (1).

\section{\$11. Produits tensoriels continus de certaines familles d'espaces hilbertiens}

Ce paragraphe s'inspire de [1]. Pour tout espace hilbertien complexe $H$ on notera $S^{n} H$ le sous-espace vectoriel fermé de l'espace hilbertien $H \otimes \cdots \otimes H$ ( $n$ fois) formé des éléments symétriques; $S^{n} H$ est aussi engendré par les éléments de la forme $x \otimes x \otimes \cdots x$ où $x \in H$ (cf. [1], §5) que nous noterons simplement $x^{n}$; on notera $S H$ la somme hilbertienne des $S^{n} H$ pour $n=0,1,2, \ldots, S^{0} H$ étant l'espace $\mathbb{C}$; notons $\varepsilon$ l'élément $(1,0,0, \ldots)$. Pour tout $x \in H$ on note $\exp x$ l'élément de $S H$ ayant pour composantes 1 dans $S^{0} H$ et $x^{n} / \sqrt{n}$ ! dans $S^{n} H$; on a

$$
(\exp x \mid \exp y)=\sum_{n=0}^{\infty}\left(x^{n} \mid y^{n}\right) / n !=\sum_{n=0}^{\infty}(x \mid y)^{n} / n !=e^{(x \mid y)} ;
$$

les éléments $\exp x(x \in H)$ forment un sous-ensemble total de $S H$ ([1], lemme 5.1); l'application $x \rightarrow \exp x$ est continue car si une suite $x_{q}$ tend vers un élément $x$ on $a$

$$
\left\|\exp x_{q}-\exp x\right\|^{2}=e^{\left|x_{q}\right|^{2}}+e^{\mid x^{2}}-2 \operatorname{Re} e^{\left(x_{q} \mid x\right)}
$$

qui tend vers 0 .

Proposition 17. Définissons I et $\mu$ comme au début du $\S 5$; soient $\left(H_{i}\right)_{i} \in I$ une famille d'espaces hilbertiens et $\Delta$ un sous-espace vectoriel de $I I H_{i}$ tel que pour tout $\left(x_{i}\right)$ et tout $\left(y_{i}\right) \in \Delta$ la fonction $i \rightarrow\left(x_{i} \mid y_{i}\right)$ soit continue 
et à support compact; soit $H$ l'espace hilbertien séparé-complété de $\Delta$ pour le produit scalaire $\left(\left(x_{i}\right) \mid\left(y_{i}\right)\right)=\int\left(x_{i} \mid y_{i}\right) d \mu(i)$; soit $\Gamma$ le sous-ensemble de II $S H_{i}$ formé des familles $\left(\lambda_{i} \exp x_{i}\right)$ où $\left(\lambda_{i}\right) \in \mathscr{K}(I)+1$ et $x=\left(x_{i}\right) \in \Delta$; la formule $(F \mid G)=\sum_{a, b \in \Gamma} F(a) \overline{G(b)} \Pi\left(a_{i} \mid b_{i}\right)$ définit un produit scalaire sur $\mathscr{K}(\Gamma)$. Il existe un isomorphisme isométrique unique de l'espace hilbertien séparé-complété de $\mathscr{K}(\Gamma)$ sur $S H$ transformant, pour tout $\left(x_{i}\right) \in \Delta$, l'image canonique de l'élément $\delta_{\left(\exp x_{i}\right)}$ de $\mathscr{K}(\Gamma)$ en l'exponentielle de l'image canonique de l'élément $\left(x_{i}\right)$ de $\Delta$.

Remarquons d'abord que la donnée d'une famille $\left(\lambda_{i} \exp x_{i}\right)$ détermine entièrement les $\lambda_{i}$ et les $x_{i}$; on notera encore $\left(x_{i}\right)$ l'image canonique de cet élément dans $H$. Soit $T$ l'application linéaire de $\mathscr{K}(T)$ dans $S H$ définie par $T(F)=\sum_{\left(\lambda_{i} \exp x_{i}\right) \in \Gamma} F\left(\left(\lambda_{i} \exp x_{i}\right)\right) \cdot \widehat{\Pi} \lambda_{i} \cdot \exp \left(x_{i}\right)$; pour $F$ et $G \in$ $\in \mathscr{K}(\Gamma)$ on a

$$
\begin{aligned}
& (T(F) \mid T(G))=\sum F\left(\left(\lambda_{i} \exp x_{i}\right)\right) \overline{G\left(\left(\lambda_{i}^{\prime} \exp x_{i}^{\prime}\right)\right)} \widehat{\Pi} \lambda_{i} \overline{\widehat{\Pi} \lambda_{i}^{\prime}}\left(\exp \left(x_{i}\right) \mid\left(\exp \left(x_{i}^{\prime}\right)\right)\right. \\
& \quad=\sum F\left(\left(\lambda_{i} \exp x_{i}\right)\right) \overline{G\left(\left(\lambda_{i}^{\prime} \exp x_{i}^{\prime}\right)\right)} \widehat{\Pi} \lambda_{i} \overline{\Pi \lambda_{i}^{\prime}} \exp \left(\int\left(x_{i} \mid y_{i}\right) d \mu(i)\right) \\
& \quad=\sum F\left(\left(\lambda_{i} \exp x_{i}\right)\right) \overline{G\left(\left(\lambda_{i}^{\prime} \exp x_{i}^{\prime}\right)\right)} \exp \left(\int \log \left(\lambda_{i} \overline{\lambda_{i}^{\prime}} e^{\left(x_{i} \mid y_{i}\right)}\right) d \mu(i)\right) \\
& \quad=\sum F\left(\left(\lambda_{i} \exp x_{i}\right)\right) \overline{G\left(\left(\lambda_{i}^{\prime} \exp x_{i}^{\prime}\right)\right)} \widehat{\Pi I}\left(\lambda_{i} \exp x_{i} \mid \lambda_{i}^{\prime} \exp x_{i}^{\prime}\right) \\
& \quad=(F \mid G)
\end{aligned}
$$

ceci prouve que la fonction $(F, G) \rightarrow(F \mid G)$ est une forme sesquilinèaire hermitienne positive sur $\mathscr{K}(T)$ et que $T$ définit une application linéaire isométrique $\bar{T}$ du séparé-complété de $\mathscr{K}(T)$ dans $S H ; \operatorname{Im} \bar{T}$ contient les éléments $\exp x$ où $x \in \Delta$; donc, par continuité, tous les éléments $\exp x$ où $x \in H$; et ceci montre que $\bar{T}$ est surjective.

\section{$\$ 12$. Produits tensoriels continus de $\mathrm{C}^{*}$-algèbres; applications à la théorie quantique des champs}

Définissons $I$ et $\mu$ comme au début du $\S 5$; soit $\left(\left(A_{i}\right),\left(e_{i}\right), \Gamma\right)$ une famille continue d'algèbres de Banach qui sont des $C^{*}$-algèbres, avec $\left(x_{i}^{*}\right) \in \Gamma$ dès que $\left(x_{i}\right) \in \Gamma$; la prop. 14 permet de considérer l'algèbre de Banach involutive $\hat{\otimes} A_{i}$ et la prop. 4 justifie la

Définition. Nous appellerons $C^{*}$-algèbre produit tensoriel continu de la famille $\left(\left(A_{i}\right),\left(e_{i}\right), \Gamma\right)$ et noterons $\check{\otimes}^{\Gamma} A_{i}$ ou $\check{\otimes} A_{i}$ la $C^{*}$-algèbre enveloppante de $\hat{\otimes} A_{i}$. On notera encore $\otimes x_{i}$ l'image canonique de $\otimes x_{i}$ dans $\check{\otimes} A_{i}$ pour tout $\left(x_{i}\right) \in \Gamma$; on ignore la norme d'un tel élément et, à vrai dire, on ignore si $\check{\otimes} A_{i}$ n'est pas réduit à 0 !.

Proposition 18. La $C^{*}$-algèbre $\check{\otimes} A_{i}$ jouit des propriétés suivantes:

(i) les éléments $\otimes x_{i}$ où $\left(x_{i}\right) \in \Gamma$ forment une partie totale de $\check{\otimes} A_{i}$ :

(ii) on $a \otimes \lambda_{i} x_{i}=\overparen{\Pi} \lambda_{i} \cdot \otimes x_{i}$ pour $\left(x_{i}\right) \in \Gamma$ et $\left(\lambda_{i}\right) \in \mathscr{K}(I)+1$;

(iii) soient $I^{0}$ une partie ouverte de $I$ vérifiant les conditions $d u \S 5$, 
$\Gamma^{0}$ l'ensemble des restrictions $\grave{a} I^{0}$ des familles $\left(x_{i}\right) \in \Gamma$ telles que $x_{i}=e_{i}$ en dehors d'un compact inclu dans $I^{0}$; il existe un morphisme unique de

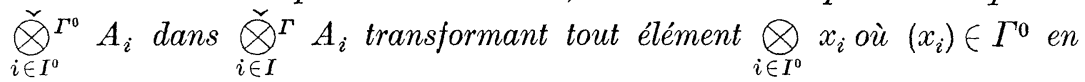
l'élément $\bigotimes_{i \in I} y_{i}$ où $y_{i}=x_{i}$ pour $i \in I^{0}$ et $y_{i}=e_{i}$ pour $i \in I^{0}$; l'image de ce morphisme est le sous-espace vectoriel fermé engendré par les éléments $\otimes y_{i}$ où $\left(y_{i}\right) \in \Gamma$ et $y_{i}=e_{i}$ en dehors d'un compact inclu dans $I^{0}$;

(iv) supposons que I soit réunion d'une famille $\left(I^{a}\right)_{a \in A}$ d'ouverts deux ̀̀ deux disjoints; notons $x^{a}$ la restriction à $I^{a}$ d'une famille quelconque $x=\left(x_{i}\right) \epsilon$ $\in \Gamma$; $\Gamma^{a}$ l'ensemble des $x^{a}$ où $\left(x_{i}\right) \in \Gamma$; on suppose en outre que pour toute famille $\left(y_{a}\right) \in \Pi \Gamma^{a}$ telle que $y_{a}=e^{a}$ pour presque tout a il existe une famille $\left(x_{i}\right) \in \Gamma$ vérifiant $y_{a}=x^{a}$ pour tout $a$; alors il existe un isomorphisme unique de $\check{\bigotimes}_{i \in I}{ }^{I} A_{i}$ sur $\bigotimes_{a \in A}\left(\bigodot_{i \in I^{a}}^{\Gamma^{a}}\right) A_{i}$ transformant $\bigotimes_{i \in I} x_{i}$ en $\bigotimes_{a \in A}\left(\bigotimes_{i \in I^{a}} x_{i}\right)$ pour tout $\left(x_{i}\right) \in \Gamma$.

Les assertions (i) et (ii) sont triviales; (iii) résulte de la remarque 4 et (iv) des prop. 4 et 8.

\section{Application à la théorie quantique des champs}

On va voir que la notion de produit tensoriel continu permet de construire une famille de $C^{*}$-algèbres $B \rightarrow \mathscr{A}(B)$ ( $B$ ouvert relativement compact de $\mathbb{R}^{4}$ ) vérifiant trois des quatre axiomes posés dans [4] pour définir la famille des algèbres d'observables locales, à savoir:

a) si $B_{1} \supset B_{2}, \mathscr{A}\left(B_{1}\right) \supset \mathscr{A}\left(B_{2}\right)$; on peut alors condsidérer les $\mathscr{A}(B)$ comme des sous-algèbres de leur limite inductive $\mathscr{A}$;

b) si $B_{1}$ et $B_{2}$ sont mutuellement de type espace [i. e. si $\left(x_{0}-y_{0}\right)^{2}-$ $-\left(x_{1}-y_{1}\right)^{2}-\left(x_{2}-y_{2}\right)^{2}-\left(x_{3}-y_{3}\right)^{2}$ est négatif pour tout $x \in B_{1}$ et tout $y \in B_{2}$ ] alors $\mathscr{A}\left(B_{1}\right)$ et $\mathscr{A}\left(B_{2}\right)$ commutent;

c) le groupe de Lorentz inhomogène $\mathscr{P}$ est représenté par des automorphismes $\alpha_{L}$ de $\mathscr{A}$, où $L \in \mathscr{P}$, de façon que $\alpha_{L}(\mathscr{A}(B))=\mathscr{A}(L B)$.

On va pour cela se placer dans le cas d'un champ scalaire neutre; notons $M$ l'espace-temps de Minkovski, ou »espace des $x \ll ; M^{*}$ l'espace dual, ou »espace des $k$ «; $m$ un nombre réel strictement positif; $C_{m}$ l'hyperboloïde dans $M^{*}$ défini par $k_{0}^{2}-k_{1}^{2}-k_{2}^{2}-k_{3}^{2}=m^{2} ; C_{m}^{+}$sa nappe supérieure $\left(k_{0}>0\right) ; \mu$ une mesure positive sur $C_{m}^{+}$invariante par le groupe de Lorentz homogène connexe $G ; v$ la mesure sur $C_{m}$ définie par $v=\mu-\check{\mu}$ ou $d \nu(k)=d \mu(k)-d \mu(-k) ; \Delta$ la distribution sur $M$ transformée de Fourier de $v$, ce qui signifie que $\Delta(f)=v(\hat{f})$ avec $\hat{f}(k)=\int e^{-i\langle k, x\rangle} f(x) d x$; $\Delta$ est invariante par $G$ et vérifie $\breve{\Delta}=-\Delta$ (parce que $\nu$ a les mêmes propriétés), d'où résulte qu'elle est nulle en dehors du cône de lumière ${ }^{1}$;

${ }^{1}$ c'est-a-dire dons le region de'finie par

$$
x_{0}^{2}-x_{1}^{2}-x_{2}^{2}-x_{3}^{2}<0
$$

Commun. math. Phys., Vol. 5 
de plus pour deux fonctions $f$ et $g$ sur $M$ on a

$$
\iint f(x) g(y) \Delta(x-y) d x d y=\int \hat{f}(-k) \hat{g}(k) d v(k) ;
$$

si en outre $f$ et $g$ sont réelles on a $\check{\hat{f}}=\overline{\hat{f}}$ et $\check{\hat{g}}=\overline{\hat{g}}$ d'où

$\iint f(x) g(y) \Delta(x-y) d x d y=\int \bar{f} \hat{g} d \mu-\int \hat{f} \bar{g} d \mu=-2 i \operatorname{Im} \int \hat{f} \overline{\hat{g}} d \mu$

et ceci est $n u l$ si les supports de $f$ et $g$ sont mutuellement de type espace.

Pour tout $k \in C_{m}^{+}$notons $E_{k}$ l'espace hilbertien $\mathbb{C}, \mathscr{A}_{k}$ son algèbre de Weyl (cf. [6]); autrement dit $\mathscr{A}_{k}$ est isomorphe à l'algèbre des opérateurs lineaires continus dans un espace hilbertien complexe de dimension infinie dénombrable et on a une application $U_{k}$ de $E_{k}$ dans l'ensemble des éléments unitaires de $\mathscr{A}_{k}$, fortement continue et vérifiant

$$
U_{k}(u) U_{k}(v) U_{k}(u)^{-1} U_{k}(v)^{-1}=e^{-2 i \operatorname{Im} u \bar{v}}
$$

de plus les $U_{k}(u)$ forment un ensemble irréductible dans $\mathscr{A}_{k}$.

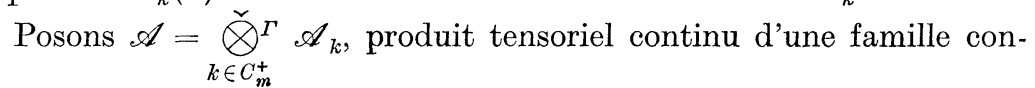
stante de $C^{*}$-algèbres; en réalité on doit modifier légèrement la définition adoptée jusqu'à maintenant de façon à pouvoir considérer des éléments de $\mathscr{A}$ de la forme $\otimes U_{k}(\hat{f}(k))$ où $f \in \mathscr{D}(M)$; on peut prendre pour $\Gamma$ l'ensemble des familles $k \rightarrow \varphi(k) U_{k}(\hat{f}(k))$ où $f \in \mathscr{D}(M)$ et où $\varphi$ est une fonction continue tendant vers 1 suffisamment vite lorsque $k \rightarrow \infty$. Pour toute fonction réelle $f \in \mathscr{D}(M)$ posons $A(f)=\otimes U_{k}(\hat{f}(k)) \in \mathscr{A}$; on a alors $A(f) A(g) A(f)^{-1} A(g)^{-1}=\otimes U_{k}(\hat{f}(k)) U_{k}(\hat{g}(k)) U_{k}(\hat{f}(k))^{-1} U_{k}(\hat{g}(k))^{-1}$

d'où, d'après (1):

$$
\begin{aligned}
& =\otimes \exp (-2 i \operatorname{Im} f(k) \overline{g(k)}) \\
& =\exp \left(-2 i \operatorname{Im} \int f \bar{g} d \mu\right)
\end{aligned}
$$

$$
A(f) A(g) A(f)^{-1} A(g)^{-1}=\exp \left(\iint f(x) g(y) \Delta(x-y) d x d y\right) .
$$

Associons à tout ouvert relativement compact $B$ de $M$ la sous- $C^{*}$-algèbre $\mathscr{A}(B)$ de $\mathscr{A}$ egendrée par les éléments $A(f)$ où supp $f \subset B$; alors l'axiome a) est vérifié trivialement et b) l'est en vertu de (2); de plus, $\Gamma$ étant choisi comme indiqué ci-dessus, $\mathscr{A}$ apparaît comme la limite inductive des $\mathscr{A}(B)$. Reste à vérifier la covariance vis à vis du groupe $\mathscr{P}$.

Soit d'abord $\Lambda$ une transformation de $M$ appartenant au groupe de Lorentz connexe homogène $G$; il en résulte une transformation $\Lambda^{\prime}$ de $M^{*}$ telle que $\left\langle\Lambda^{\prime} k, x\right\rangle=\left\langle k, \Lambda^{-1} x\right\rangle ; \Lambda^{\prime}$ induit un homéomorphisme de $C_{m}^{+}$ conservant $\mu$, d'où un automorphisme $\alpha_{\Lambda}$ de $\mathscr{A}$ transformant tout élément $\otimes b_{k}$ en $\otimes c_{k}$ où $c_{k}=b_{\Lambda^{\prime-1} k} ;$ pour toute $f \in \mathscr{D}(M)$ posons $\left(\Lambda f(x)=f\left(\Lambda^{-1} x\right)\right.$; alors on vérifie aisément que

$$
A(\Lambda f)=\alpha_{\Lambda}(A(f)) \text {. }
$$

Considérons maintenant une translation de $M: x \rightarrow x+a$; posons $(a f)(x)=f(x-a)$; pour tout $k \in C_{m}^{+}$on a un automorphisme de $E_{k}$ : $u \rightarrow e^{-i\langle k, a\rangle} \cdot u$; d'où un automorphisme $\alpha_{a, k}$ de $\mathscr{A}_{k}$ tel que $\alpha_{a, k}\left(U_{k}(u)\right)$ $=U_{k}\left(e^{-i\langle k, a\rangle} \cdot u\right)$ pour tout $u \in E_{k}$; soit $\alpha_{a}$ l'automorphisme de $\mathscr{A}$, 
produit tensoriel des $\alpha_{a, k}: \alpha_{a}\left(\otimes b_{k}\right)=\otimes \alpha_{a, k}\left(b_{k}\right)$; alors on a facilement

$$
A(a f)=\alpha_{a}(A(f)) \text {. }
$$

Il est facile de vérifier que $\alpha_{\Lambda} \alpha_{a} \alpha_{\Lambda}^{-1}=\alpha_{\Lambda a}$, ce qui permet de définir un morphisme $L \rightarrow \alpha_{I}$ du groupe $\mathscr{P}$ dans le groupe des automorphismes de $\mathscr{A} ;(3)$ et (4) montrent que $A(L f)=\alpha_{L}(A(f))$; d'où l'axiome c).

Enfin on doit pouvoir interpréter la représentation classique de Fok comme produit tensoriel continue des représentations de Schrödinger des diverses algèbres $\mathscr{A}_{k}$; indiquons le principe du raisonnement (qui en fait se heurte à un certain nombres de difficultés techniques non encore surmontées): chaque $\mathscr{A}_{k}$ admet une représentation irréductible $\pi_{k}$ (qui en est d'ailleurs la seule représentation irréductible "normale«) dans l'espace $S E_{k}$ (cf. $\S 11$ ), associée à un état $\varphi_{k}$ de $\mathscr{A}_{k}$ tel que $\varphi_{k}\left(U_{k}(u)\right)$ $=e^{-\frac{1}{2}|u|^{2}}$ pour tout $u \in E_{k}$; posons $\varphi=\otimes \varphi_{k}$ (en admettant que ce soit effectivement un état!); alors on aura

$$
\varphi(A(f))=\widehat{I I} e^{-\frac{1}{2}|\hat{f}(k)|^{2}}=e^{-\frac{1}{2} \int|\hat{f}|^{2} d_{\mu}}
$$

de plus on peut raisonnablement penser que la représentation de $\mathscr{A}$ associée à $\varphi$ opère dans l'espace hilbertien $\otimes S E_{k}$, lequel, d'après la prop. 17, devrait être isomorphe à $S\left(\int^{\oplus} E_{k} d \mu(k)\right)=S\left(L^{2}\left(C_{m}^{+}, \mu\right)\right)$-espace hilbertien dans lequel opère effectivement la représentation classique de Fok.

Ajoutons pour terminer que plusieurs conversations avec D. KASTLER nous ont guidé dans la rédaction de ce dernier paragraphe.

Signalons aussi qu'on peut modifier la construction précédente pour faire opérer dans $\mathscr{A}$ le groupe de Lorentz complet; et qu'on peut faire une construction analogue dans le cas du champ de Maxwell.

Ajouté en épreuves. On a repris et un peu amélioré tout ce travail, mais surtout le dernier paragraphe, dans un cours fait à l'Université de Strasbourg à l'occasion de la R.C.P. $\mathrm{n}^{\circ} 25$, et qui sera publié ultérieurement.

\section{Bibliographie}

1. Araki, H., and E. J. Woods: Complete Boolean algebras of type I factors. Publ. Res. Inst. Math. Sci. Kyoto Univ. 2, 157-242 (1966).

2. Dixmier, J., and A. Douady: Champs continus d'espaces hilbertiens et de $C^{*}$ algèbres. Bull. Soc. Math. France 91, 227-284 (1963).

3. Guichardet, A.: Produits tensoriels infinis et représentations des relations d'anticommutation. Ann. Sci. Ecole. Norm. Super. 83, 1-52 (1966).

4. HAAG, R., and D. KASTLER: An algebraic approach to quantum field theory. J. Math. Phys. 5, 848-861 (1964).

5. Mrchael, E.: Continuous selections; I. Ann. Math. 63, 361-382 (1956).

6. Segal, I. E.: Mathematical characterization of the physical vacuum for a linear Bose-Einstein field. Illinois J. Math. 6, 500-523 (1962).

7. Zeller-Meier, G.: Produits croisés d'une $C^{*}$-algèbre par un groupe d'automorphismes. C.R. Acad. Sci. Paris 263, 20-23 (1966). 